Article

\title{
Analysis of the Induction and Wake Evolution of an Offshore Floating Wind Turbine
}

\section{Thomas Sebastian * and Matthew Lackner}

Department of Mechanical and Industrial Engineering, University of Massachusetts, 160 Governors Drive, Amherst, MA 01003-9265, USA; E-Mail: lackner@ecs.umass.edu

* Author to whom correspondence should be addressed; E-Mail: tommy.sebastian@ gmail.com.

Received: 3 February 2012; in revised form: 29 March 2012 / Accepted: 9 April 2012 /

Published: 17 April 2012

\begin{abstract}
The degrees-of-freedom associated with offshore floating wind turbines (OFWTs) result in a more dynamic flow field. The resulting aerodynamic loads may be significantly influenced by these motions via perturbations in the evolving wake. This is of great interest in terms of OFWT design, placement and simulation. This study presents free vortex wake method (FVM) simulations of the NREL 5-MW wind turbine of a variety of platforms, operating in a range of wind speeds synthesized platform motion time series. Motion-induced wake perturbations are observed to affect induction. Transitions between windmill and propeller states are also observed.
\end{abstract}

Keywords: offshore floating wind turbine; free vortex wake method; wake evolution

\section{Nomenclature}

$\begin{array}{llll}1 P & \text { Once per revolution } & H_{s} & \text { Significant wave height } \\ A & \text { Amplitude } & K & \text { Gain } \\ a & \text { Axial induction } & n & \text { Core model index } \\ c & \text { Chord length } & R & \text { Radial location along span } \\ C_{l} & \text { Lift coefficient } & s & \text { Laplace variable } \\ C_{T} & \text { Thrust coefficient } & t & \text { Time } \\ D & \text { Rotor diameter } & T_{p} & \text { Peak spectral period } \\ f & \text { Frequency } & U & \text { Velocity }\end{array}$




$\begin{array}{llll}X & \text { Synthesized time series } & \phi & \text { Phase angle } \\ \alpha & \text { Angle of attack } & \psi & \text { Azimuth angle } \\ \beta & \text { Sideslip angle } & \sigma & \text { Standard deviation } \\ \Gamma & \text { Circulation strength } & \tau & \text { Time constant } \\ \lambda & \text { Tip speed ratio } & \theta & \text { Platform angular position } \\ \lambda_{D} & \text { Advection wavelength } & \theta_{\text {twist }} & \text { Blade twist angle } \\ \mu & \text { Mean } & \zeta & \text { Wake age } \\ \Omega & \text { Rotor speed } & & \end{array}$

\section{Introduction}

Most studies on the design, dynamics, and performance of offshore floating wind turbines (OFWTs) implicitly assume that commonly-used aerodynamic analysis methods are sufficient. Sebastian and Lackner [1] demonstrated that this assumption may not be entirely correct and that the additional degrees-of-freedom (DOFs) associated with OFWT platform motions (Figure 1) results in aerodynamic unsteadiness that exceeds the unsteadiness experienced by onshore and offshore fixed bottom systems.

Figure 1. Offshore floating wind turbine platform DOFs.

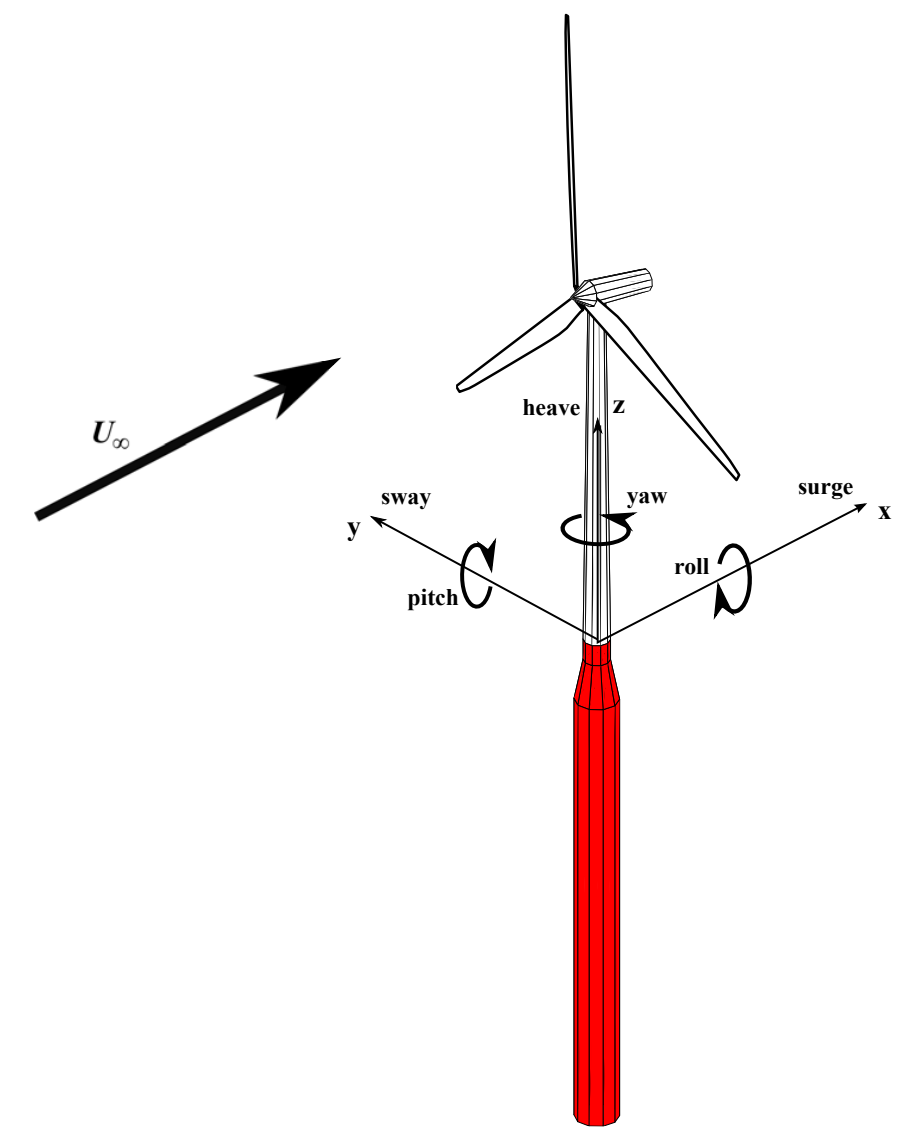

These platform DOFs generate an effective flow field velocity perturbation, $U_{\text {platform }}$, given by Equation (1), 


$$
\begin{aligned}
\mathbf{U}_{\text {platform }}=( & \left.U_{\text {surge }}+\dot{\theta}_{\text {pitch }} z-\dot{\theta}_{\text {yaw }} y\right) \hat{\mathbf{i}}+\left(U_{\text {sway }}+\dot{\theta}_{\text {yaw }} x-\dot{\theta}_{\text {roll }} z\right) \hat{\mathbf{j}} \\
& +\left(U_{\text {heave }}+\dot{\theta}_{\text {roll }} y-\dot{\theta}_{\text {pitch }} x\right) \hat{\mathbf{k}}
\end{aligned}
$$

where $x, y$, and $z$ are the coordinates of a point in the flow field in the rotor reference frame. This additional velocity contribution is what sets the aerodynamic analysis of OFWTs apart from conventional wind turbines and may necessitate changes in turbine rotor design for floating configurations, modifications to current spacing and grid arrangements for wind farms, and adjustments to blade pitch and generator torque controller schemes. This study investigates the effect of platform motions on the aerodynamic loading and wake evolution of different OFWT configurations under a range of operating conditions using a free vortex wake method.

\section{Methodology}

\subsection{NREL 5-MW Turbine and Platforms}

There are very little publicly available data on operational OFWTs, because of the limited number of deployed systems and their proprietary nature. In lieu of experimental data, computer simulations may be used to generate time series of platform motions, using realistically-scaled turbine and platform properties and user-defined load cases as inputs. NREL identified the need for a standardized reference wind turbine to provide realistic input values for offshore wind energy analysis and developed the "NREL offshore 5-MW baseline wind turbine" [2], which is identified in this study as the NREL 5-MW wind turbine. The NREL 5-MW turbine, based on the REpower 5M machine, is a conventional three-bladed upwind turbine. A summary of the physical properties and rated/off-rated operating conditions for the NREL 5-MW turbine is given in Table 1. The blade geometry, with airfoil types defined along the span, is illustrated by Figure 2.

Table 1. Properties of the NREL 5-MW baseline wind turbine [2].

\begin{tabular}{lr}
\hline Rating & $\mathbf{5 ~ M W}$ \\
\hline Rotor Orientation, Configuration & Upwind, 3 Blades \\
Control & Variable Speed, Collective Pitch \\
Rotor, Hub Diameter & $126 \mathrm{~m}, 3 \mathrm{~m}$ \\
Hub Height & $90 \mathrm{~m}$ \\
Cut-In, Rated. Cut-Out Wind Speed & $3 \mathrm{~ms}^{-1}, 11.4 \mathrm{~ms}^{-1}, 25 \mathrm{~ms}^{-1}$ \\
Cut-In, Rated Rotor Speed & $6.9 \mathrm{rpm}, 12.1 \mathrm{rpm}^{-1}$ \\
Rated Tip Speed & $80 \mathrm{~ms}^{-1}$ \\
Overhang, Shaft Tilt, Precone & $5 \mathrm{~m}, 5^{\circ}, 2.5^{\circ}$ \\
Rotor Mass & $110,000 \mathrm{~kg}$ \\
Nacelle Mass & $240,000 \mathrm{~kg}$ \\
Tower Mass & $347,460 \mathrm{~kg}$ \\
\hline
\end{tabular}


Figure 2. NREL 5-MW rotor blade geometry.

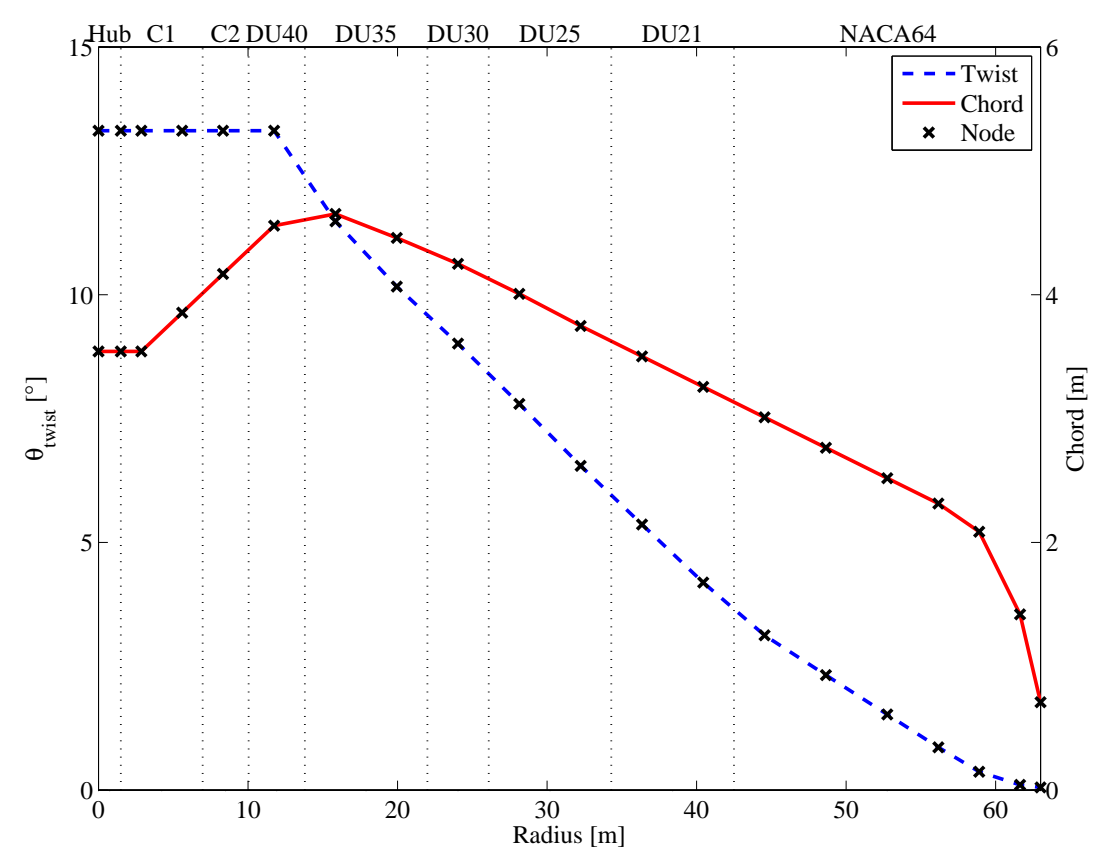

A number of floating platforms have been designed for integration with offshore wind turbines, drawing on experiences in the oil and gas industry. The three main platform classes-barge, spar-buoy, and tension leg platform (TLP) — have been scaled to complement the NREL 5-MW turbine, thereby facilitating realistic simulations. The ITI Energy barge [3] was developed by the Department of Naval Architecture and Marine Engineering at the Universities of Glasgow and Strathclyde under contract by ITI Energy. The OC3-Hywind spar-buoy [4] was developed under the International Energy Agency (IEA) within the Offshore Code Comparison Collaboration (OC3) project and is based on the StatoilHydro "Hywind" spar-buoy. The MIT/NREL TLP [5] design is the product of a parametric optimization process conducted at the Massachusetts Institute of Technology (MIT), incorporating small modifications to accommodate the NREL 5-MW turbine. The physical properties of these platforms are summarized in Table 2.

Table 2. NREL 5-MW platform properties [7].

\section{Properties}

Effective diameter $[\mathrm{m}]$

Draft $[\mathrm{m}]$

Water depth [m]

Displaced volume of water $\left[\mathrm{m}^{3}\right]$

Mass $\left[10^{3} \mathrm{~kg}\right]$

CM location below SWL [m]

Roll inertia about CM $\left[10^{6} \mathrm{~kg} \mathrm{~m}^{3}\right]$

Pitch inertial about $\mathrm{CM}\left[10^{6} \mathrm{~kg} \mathrm{~m}^{3}\right]$

Yaw inertia about $\mathrm{CM}\left[10^{6} \mathrm{~kg} \mathrm{~m}^{3}\right]$

OC3-Hywind spar-buoy ITI Energy barge MIT/NREL TLP

6.5 to 9.5

120

320

$8,029.21$

$7,466.33$

89.92

4, 229.23

$4,229.23$

164.23

$45.14(40 \times 40)$

4

250

6, 000.0

5, 452

0.28

726.9

726.9

1, 453.9
18

47.89

200

$12,179.6$

8, 600.41

40.61

571.624

571.624

361.408 


\subsection{FAST}

A number of aero-hydrodynamic codes have been developed to predict the dynamics and performance of OFWTs. FAST, an example of such a code, is a freely available, comprehensive, aeroelastic simulator developed by NREL [8]. FAST is capable of modeling the dynamics and performance of a wind turbine system, given wind data and the physical properties of the system. Aerodynamic loads are computed by the AeroDyn subroutines, which apply momentum balance assumptions via blade element momentum (BEM) and generalized dynamic wake (GDW) approaches [9]. It has been evaluated by Germanischer Lloyd WindEnergie and found suitable for "the calculation of onshore wind turbine loads for design and certification" [10]. The HydroDyn [11] subroutines compute "the restoring contributions of buoyancy and waterplane area from hydrostatics, the viscous drag contributions from Morison's equation, the added mass and damping contributions from wave radiation, including free surface memory effects, and the incident wave excitation from scattering in regular or irregular seas". Mooring system loads are also calculated. In the absence of experimental OFWT data, FAST is used to synthesize OFWT performance and structural response time series. These time series may be used as inputs for higher-fidelity analysis tools, such as the Wake Induced Dynamics Simulator discussed in the next section.

\section{3. $W I n D S$}

Momentum balance approaches are conceptually simple, but rely on a number of ad hoc, empirically-derived corrections. Recognizing that the external flow of a wind turbine is nominally inviscid, incompressible, and irrotational permits the use of potential flow methods. These assumptions are global, physically consistent descriptions of the flow rather than experimentally limited extrapolations. Free vortex wake methods (FVM) are a subset of potential flow and have been in use for a number of decades. This study uses the Wake Induced Dynamics Simulator (WInDS) code developed by Sebastian and Lackner [12], a lifting-line theory (LLT) based FVM developed at the University of Massachusetts at Amherst for OFWTs and validated via comparison to analytical models and experimental data. Under potential flow assumptions, vortex filament nodes move as Lagrangian markers with the local fluid flow. The advection equation that describes the motion of the vortex filament nodes is given by Equation (2),

$$
\frac{d \mathbf{x}}{d t}=\mathbf{U}
$$

where $\mathbf{U}$ is the velocity of the local fluid in the rotor reference frame. The azimuthal rotor position, $\psi$, and wake age, $\zeta$, may be used to define nodal position (Equation (3)),

$$
\frac{d \mathbf{x}}{d t}=\frac{\partial \psi}{\partial t} \frac{\partial \mathbf{x}}{\partial \psi}+\frac{\partial \zeta}{\partial t} \frac{\partial \mathbf{x}}{\partial \zeta}=\Omega\left(\frac{\partial \mathbf{x}}{\partial \psi}+\frac{\partial \mathbf{x}}{\partial \zeta}\right)=\mathbf{U}
$$

where $\Omega$ is the rotor speed and the contributions to $\mathbf{U}$ are given as

$$
\mathbf{U}=\mathbf{U}_{\infty}+\mathbf{U}_{\text {induced }}+\mathbf{U}_{\text {platform }}
$$

where $U_{\infty}$ and $U_{\text {induced }}$ denote free stream and induced velocity vectors, respectively. Recall (Equation (1)) that the additional perturbation velocity, $\mathbf{U}_{\text {platform }}$, acts as an additional forcing term 
on the advection of the vortex filament nodes. The wake lattice will grow with each time step as the wake nodes are numerically advected, illustrated by Figure 3.

Figure 3. Vortex lattice wake structure, illustrating wake evolution between time steps.

(a) $t_{0} ;$ (b) $t_{1} ;$ (c) $t_{2} ;$ (d) $t_{3}$.

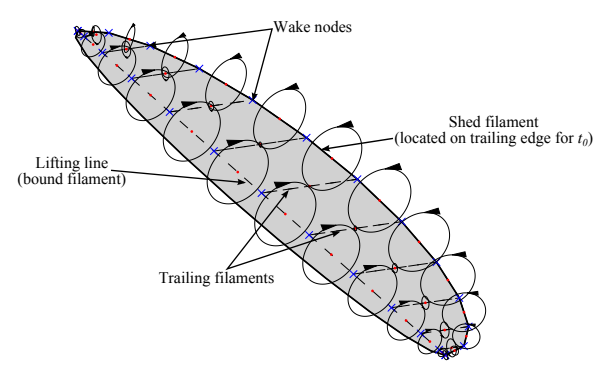

(a)

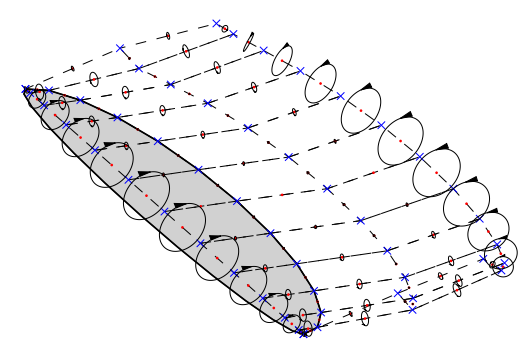

(c)

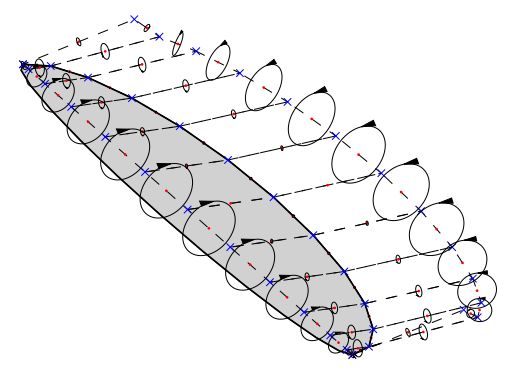

(b)

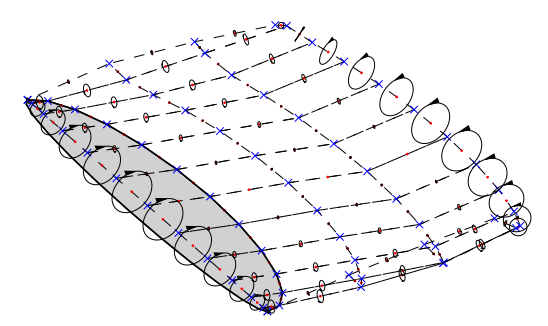

(d)

Algorithm 1 outlines the implementation of the WInDS code.

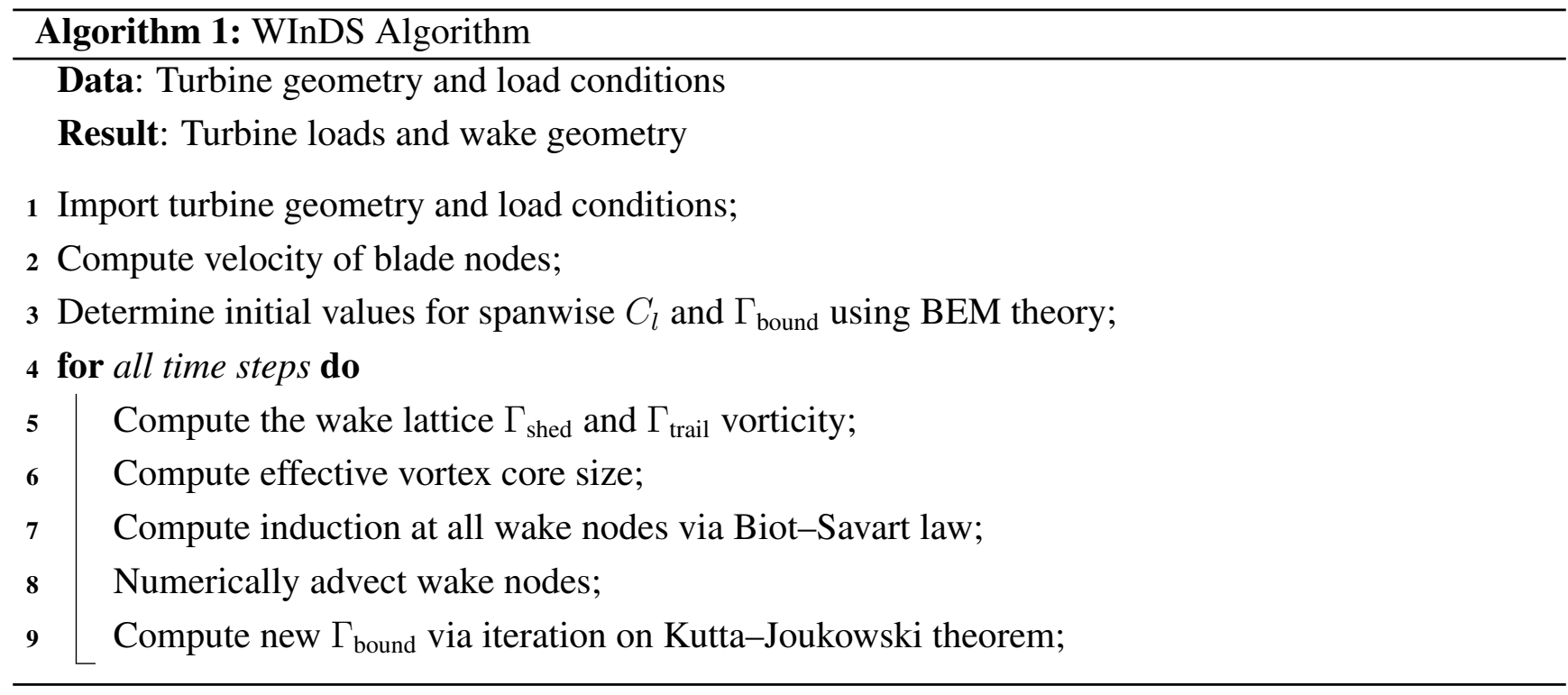

\subsection{Simulation Settings and Test Cases}

WInDS does not currently couple aerodynamic loading with platform dynamics. As was mentioned earlier, FAST may be used to generate realistic time series of platform motions; these time series may then be imported by WInDS. 


\subsubsection{FAST Settings}

Table 3 summarizes the load cases for the NREL 5-MW monopile, barge, spar-buoy, and TLP systems in terms of wind speed and sea state, simulated via FAST.

Table 3. Wind and sea state definitions for FAST simulations of the NREL 5-MW turbine.

\begin{tabular}{lccccc}
\hline Rotor Mode & $\boldsymbol{U}_{\boldsymbol{\infty}}\left[\mathbf{m s}^{-\mathbf{1}}\right]$ & $\boldsymbol{\Omega}[\mathbf{r p m}]$ & $\boldsymbol{\lambda}$ & $\boldsymbol{H}_{\boldsymbol{s}}[\mathbf{m}]$ & $\boldsymbol{T}_{\boldsymbol{p}}[\mathbf{s}]$ \\
\hline Below-rated & 6.00 & 8.76 & 9.63 & 1.83 & 12.72 \\
Rated & 11.40 & 12.10 & 7.00 & 2.54 & 13.35 \\
Above-rated & 18.00 & 12.10 & 4.43 & 4.09 & 15.33 \\
\hline
\end{tabular}

In addition to these operating conditions, the FAST simulations were conducted with the following settings:

- The six platform DOFs and the tower fore-aft and side-side DOFs were switched on to yield realistic modal responses.

- An equilibrium BEM inflow model was used. Use of a dynamic inflow model would introduce a time lag to the inflow and load response.

- Blade pitch and generator torque controllers were switched on.

- Dynamic stall is switched off.

- Free stream wind was defined as constant, unidirectional, and without shear.

- Wind and wave data from BMT ARGOSS [3] were used to define the sea state in terms of significant wave height, $H_{s}$, and peak spectral period, $T_{p}$. The Joint North Sea Wave Project (JONSWAP) spectrum [6] was used to generate a simulated time series.

Time domain simulations were performed for each of the cases described in Table 3, with each simulation lasting $800 \mathrm{~s}$. The outputs generated by the initial $300 \mathrm{~s}$ of each simulation were omitted in the analysis. Note that these FAST simulations were conduced using an equilibrium BEM model, but were used to generate time series for WInDS, which is an unsteady aerodynamic model. Inclusion of unsteady aerodynamics in the FAST simulations would make it difficult to separate the contributions to the platform motions deriving from wave loading and model-based rotor loading, which may or may not be applicable to these cases. These load cases and settings represent a compromise between simulation realism and the ability to extract system-level understanding of the underlying rotor-wake dynamics.

\subsubsection{Single and Multiple-DOF WInDS Test Cases}

Because many of the platform modes share similar spectral peaks, it becomes difficult to discern the aerodynamic contributions of one mode from those of another. In an earlier study, Sebastian and Lackner [1] determined that the majority of the energy in each of the platform modes is attributable to two primary frequencies, generally associated with the sea state and the $1 \mathrm{P}$ rotor rate. Therefore, rather than to import the FAST-simulated time series directly into WInDS, sinusoids of the form given by Equation (5), limited to the two peak frequencies of each platform mode, were fitted to each of 
the FAST-simulated platform responses and used to generate the platform motion time series used by WInDS, resulting in a set of synthesized time series, $X$.

$$
X=X_{0}+A_{1} \sin \left(2 \pi f_{1}+\phi_{1}\right)+A_{2} \sin \left(2 \pi f_{2}+\phi_{2}\right)
$$

This approach is conservative as the fitted platform motion has lower variability than the original FAST-simulated time series. Sebastian and Lackner [1] demonstrated that OFWTs are more likely to generate unsteady aerodynamic loading when compared to a monopile. This earlier work also identified the OFWT modes most likely to generate unsteady loading, which informs the selected test cases in this study. Table 4 outlines the selected single-DOF test cases in terms of the variables in Equation (5).

Table 4. Single-DOF analysis cases.

\begin{tabular}{lcccccccccc}
\hline Platform Type & $\boldsymbol{U}_{\boldsymbol{\infty}}\left[\mathbf{m s}^{-\mathbf{1}}\right]$ & $\boldsymbol{\theta}_{\text {blade }}$ & Mode & \multicolumn{1}{c}{$\boldsymbol{X}_{\mathbf{0}}$} & \multicolumn{1}{c}{$\boldsymbol{A}_{\mathbf{1}}$} & $\boldsymbol{f}_{\mathbf{1}}[\mathbf{H z}]$ & $\boldsymbol{\phi}_{\mathbf{1}}[\mathbf{r a d}]$ & $\boldsymbol{A}_{\mathbf{2}}$ & $\boldsymbol{f}_{\mathbf{2}}[\mathbf{H z}]$ & $\boldsymbol{\phi}_{\mathbf{2}}[\mathbf{r a d}]$ \\
\hline ITI Energy barge & 6.0 & 0.0 & Surge & 13.602 & 0.725 & 0.007 & -1.163 & -0.442 & 0.078 & 2.609 \\
ITI Energy barge & 6.0 & 0.0 & Heave & -0.130 & 0.318 & 0.078 & 1.303 & 0.254 & 0.108 & 2.702 \\
ITI Energy barge & 6.0 & 0.0 & Pitch & 0.591 & 1.475 & 0.078 & -0.066 & 1.630 & 0.083 & 1.816 \\
OC3-Hywind spar-buoy & 6.0 & 0.0 & Pitch & 1.580 & -0.084 & 0.066 & 1.930 & -0.116 & 0.077 & 3.113 \\
OC3-Hywind spar-buoy & 6.0 & 0.0 & Yaw & -0.021 & 0.091 & 0.108 & 1.983 & -0.036 & 0.120 & 3.429 \\
MIT/NREL TLP & 6.0 & 0.0 & Surge & 1.206 & 0.436 & 0.016 & -0.831 & -0.222 & 0.077 & 3.018 \\
\hline ITI Energy barge & 11.4 & 0.0 & Pitch & 1.722 & -0.637 & 0.065 & -0.381 & 1.677 & 0.077 & 1.835 \\
\hline ITI Energy barge & 18.0 & 15.0 & Pitch & 0.939 & 1.518 & 0.066 & 2.132 & 2.979 & 0.078 & 6.863 \\
OC3-Hywind spar-buoy & 18.0 & 15.0 & Pitch & 3.324 & 11.961 & 0.029 & 0.420 & 0.000 & 0.000 & 0.000 \\
OC3-Hywind spar-buoy & 18.0 & 15.0 & Yaw & -0.222 & 2.000 & 0.029 & -0.359 & 3.185 & 0.058 & 3.385 \\
\hline
\end{tabular}

In addition to the three (below-rated, rated, and above-rated) monopile cases and the single-DOF cases, three multiple-DOF simulations were also conducted, combining the properties listed in Table 4:

- The ITI Energy barge under below-rated operating conditions with platform surge, heave, and pitch DOFs.

- The OC3-Hywind spar-buoy under below-rated operating conditions with platform pitch and yaw DOFs.

- The OC3-Hywind spar-buoy under above-rated operating conditions with platform pitch and yaw DOFs.

\subsubsection{WInDS Settings}

The NREL 5-MW wind turbine with both the monopile and floating platforms was simulated in WInDS using a $2.67 \mathrm{GHz}$ Intel Core i5 CPU 750 with $8 \mathrm{~GB}$ of RAM running Windows 7 Professional (64-bit) with MATLAB Version 7.12.0.635. After some initial cases were run, a flat plate airfoil was selected for use across the entire blade span instead of the set of airfoils listed in Figure 2. The initial cases indicated an artificial sensitivity to blade discretization when using the full complement of airfoils. Flat plate airfoils represent a compromise between simulation and model fidelity. However, as this would yield artificially low loading for above-rated cases, an offset of $C_{l_{0}}=0.4$ was applied to the flat 
plate assumption for these cases. Blades were pitched to feather by $15^{\circ}$ for above-rated cases to shed excess load; otherwise, blade pitch was set to $0^{\circ}$. All simulations used the second-order Runge-Kutta (RK2) numerical integration scheme to convect the wake downstream, modeled as a freely deforming lattice of vortex filaments. Cores were modeled using the Vatistas vortex model with $n=1$, thereby approximating the Scully vortex model [12]. All cases were discretized at 5 points per second for $120 \mathrm{~s}$ with platform motions beginning after $10 \mathrm{~s}$, corresponding with $\Delta \psi$ values of $10.5^{\circ}$ for the below-rated and rated cases and $14.5^{\circ}$ for the above-rated case, which is sufficient for second-order accuracy [13].

\section{Wake Evolution and Induction}

Equation (1) indicates that platform motions will result in velocity perturbations in the wake. The wake induces loads on the rotor, so one would expect perturbations in the wake to manifest as unsteady loading on the rotor. Wake self-induction may also be affected by these velocity perturbations, which will affect how the wake evolves downstream. Note that the resulting coupled dynamics between wake evolution and rotor loading are highly nonlinear.

\subsection{Wake Evolution}

Figures 4 and 5 present the WInDS-simulated wake for the ITI Energy barge and OC3-Hywind sparbuoy test cases. The colors differentiate each of the blades' wake structure contributions, with the tip trailing filament highlighted throughout the wake for clarity. The transparency in the wake is scaled by the influence of the wake at the rotor; wake sections that induce larger relative velocities at the rotor are more opaque than sections with lower induction.

Figure 4. WInDS-simulated wake for the NREL-5MW turbine + ITI Energy barge. (a) Surge, below-rated; (b) Heave, below-rated; (c) Pitch, below-rated; (d) Pitch, rated; (e) Pitch, above-rated.

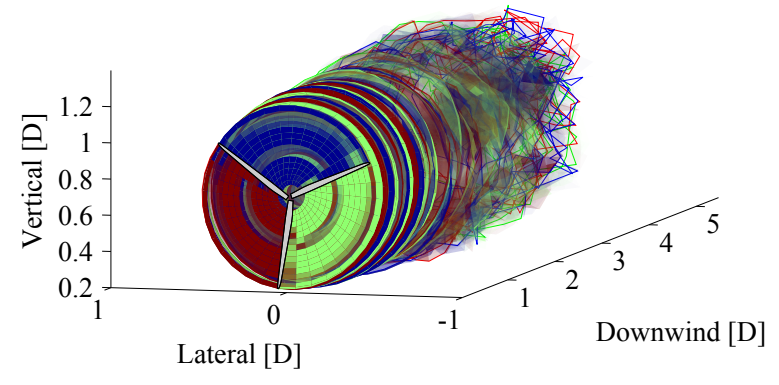

(a)

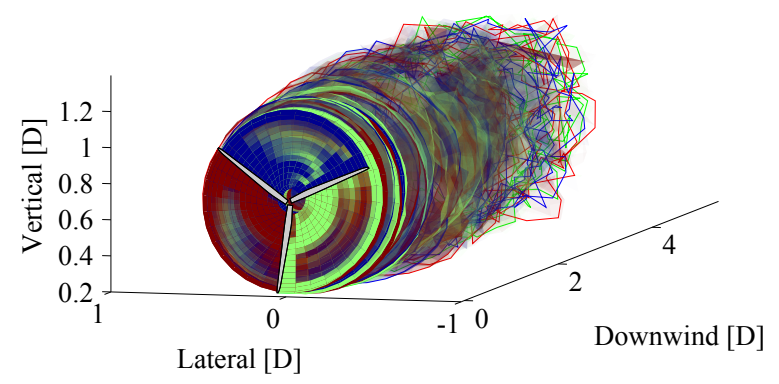

(c)

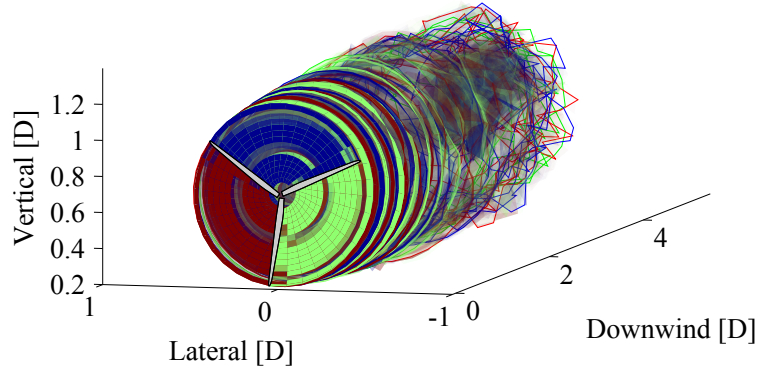

(b)

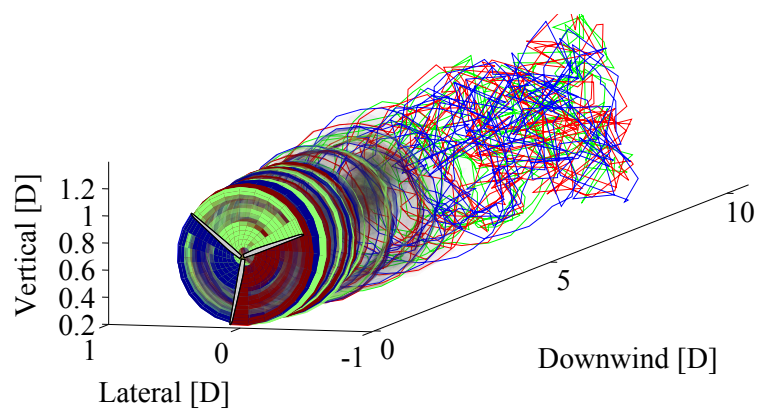

(d) 
Figure 4. Cont.

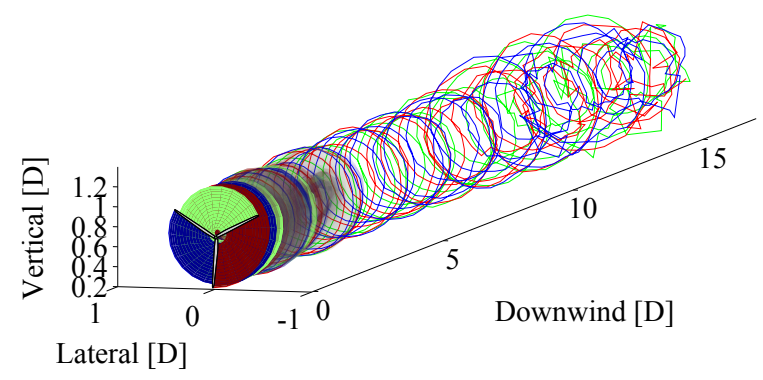

(e)

Figure 5. WInDS-simulated wake for the NREL-5MW turbine + OC3-Hywind spar-buoy. (a) Pitch, below-rated; (b) Yaw, below-rated; (c) Pitch, above-rated; (d) Yaw, above-rated.

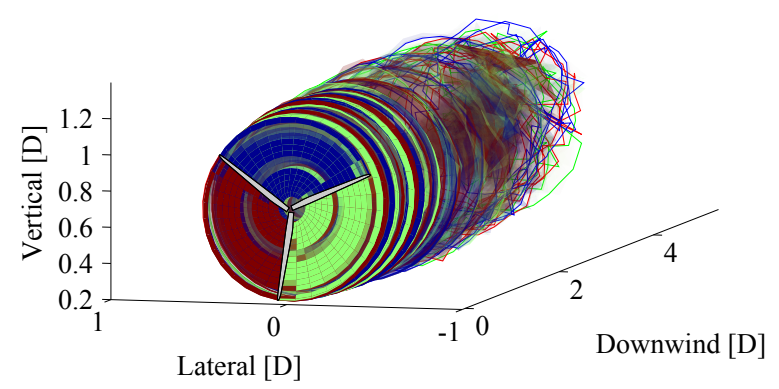

(a)

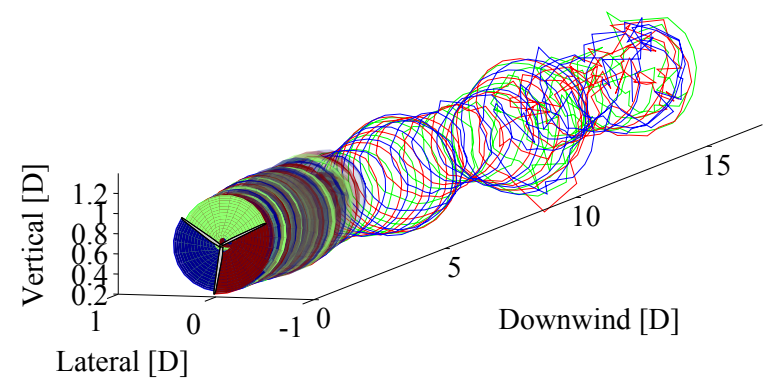

(c)

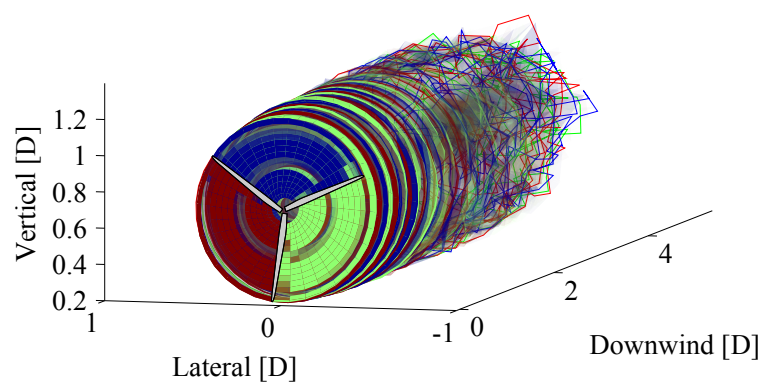

(b)

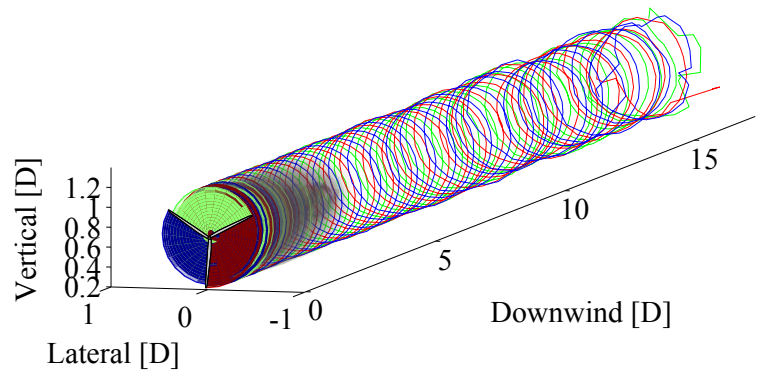

(d)

Physical stability of the wake is observed in the cohesiveness of the wake as it advects downstream. A stable wake will maintain a generally helicoid structure with minimal variation in the parameterized (with respect to the helicoid wake structure) wake nodes as a function of wake age. The physical stability of the wakes generated by the ITI Energy barge test cases decreases as the tip speed ratio increases, as illustrated via comparison of Figure $4 \mathrm{c}-\mathrm{e}$. Note that the helicoid structure breaks down and that the wake nodes appear to diverge from this generally helicoid wake structure as a function of wake age. At lower free stream wind speeds, the vortex filaments convect downstream at a slower rate, resulting in a more prominent wake self-induction than at higher wind speeds. Comparing Figure $4 \mathrm{a}-\mathrm{c}$ indicates that wake stability is somewhat insensitive to platform mode at high tip speed ratios-all modes are unstable for high tip speed ratios - though platform pitch yields a slightly more unstable wake. Compression of vortex filaments in the wake, due to platform pitching motions, is clearly shown in Figure 4e and 
manifests as an accordion-like train of lobes advecting downstream. Also note that there is some transparency in the wake in the rotor plane at the midspan of the blade, indicating that induction is greater near the root and tip of the blades; this is attributable to wake roll-up and the resulting concentration of vorticity.

As with the ITI Energy barge, Figure 5 indicates that wake stability is inversely related to tip speed ratio for the below-rated and above-rated OC3-Hywind spar-buoy cases. Figure 5d shows that the wake associated with the platform yaw mode is significantly more stable than the wake associated with the platform pitch mode, and remains stable as far out as 15 rotor diameters (D). As with the pitching barge in Figure 4e, motion-induced compression lobes are observed for the pitching spar-buoy in Figure 5c. For all cases, the wake maintains a coherent helicoidal shape, with prominent tip vortex roll-up structures fully formed within one rotor diameter (1D).

\subsection{Wake Induction at the Rotor}

Figure 6 compares the WInDS-simulated wake for the monopile under all tested operating conditions and the MIT/NREL TLP operating in below-rated winds with a platform surging motion.

Figure 6. Side view of WInDS-simulated wake for the NREL-5MW turbine with monopile and MIT/NREL TLP. (a) Surge, MIT/NREL TLP, below-rated; (b) Monopile, below-rated; (c) Monopile, rated; (d) Monopile, above-rated.

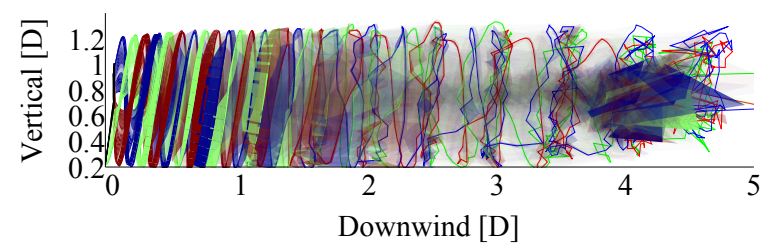

(a)

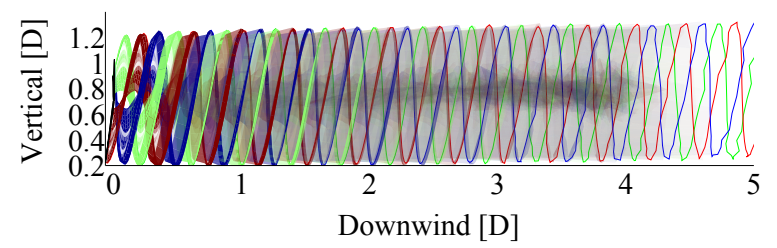

(c)

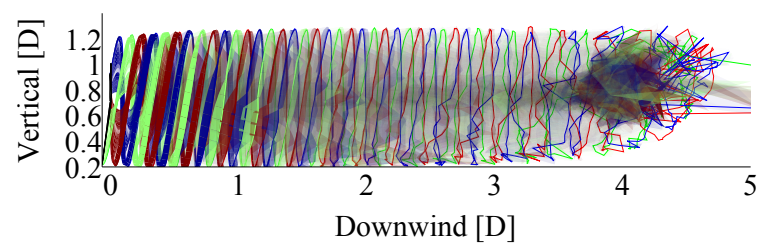

(b)

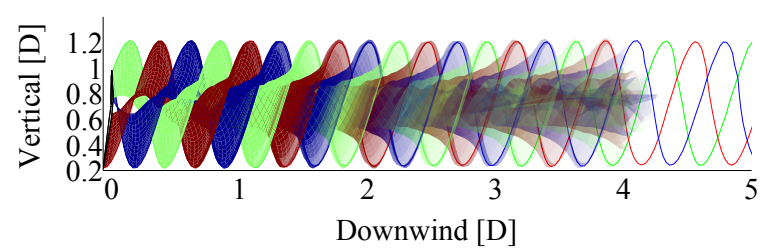

(d)

As the wind speed increases, the wake convects downstream faster, carrying along with it the starting vortex. Once the starting vortex has convected downstream a sufficient distance, its influence on the rotor becomes negligible; barring any changes in operation, the rotor operates at a steady state. From the wake transparency in Figure $6 a, b$, it is clear that the starting vortex has a significant influence on the induced flow at the rotor, even at 4 rotor diameters downstream. Figure $6 \mathrm{c}, \mathrm{d}$ demonstrate that once the starting vortex has convected further downstream, the influence of the steady-state wake becomes negligible beyond 4D downstream.

The wake induction at the rotor may be computed as a fraction of the overall induced velocity at the rotor and defined as a function of the downstream distance of the filaments, indicating what percentage 
of rotor induction is attributable to downstream wake elements. Integrating the resulting curve yields the cumulative influence of the wake as a function of downstream distance. Analogous to a Pareto plot, commonly-used in statistical analysis, these curves highlight the point at which wake contributions are negligible.

Figure 7a provides an example for the ITI Energy barge under platform surge, heave, and pitch for below-rated operating conditions for $t=120 \mathrm{~s}$.

Figure 7. Wake induced velocity at the lifting-line for the NREL-5MW turbine + ITI Energy barge under platform surge, heave, and pitch for below-rated operating conditions.

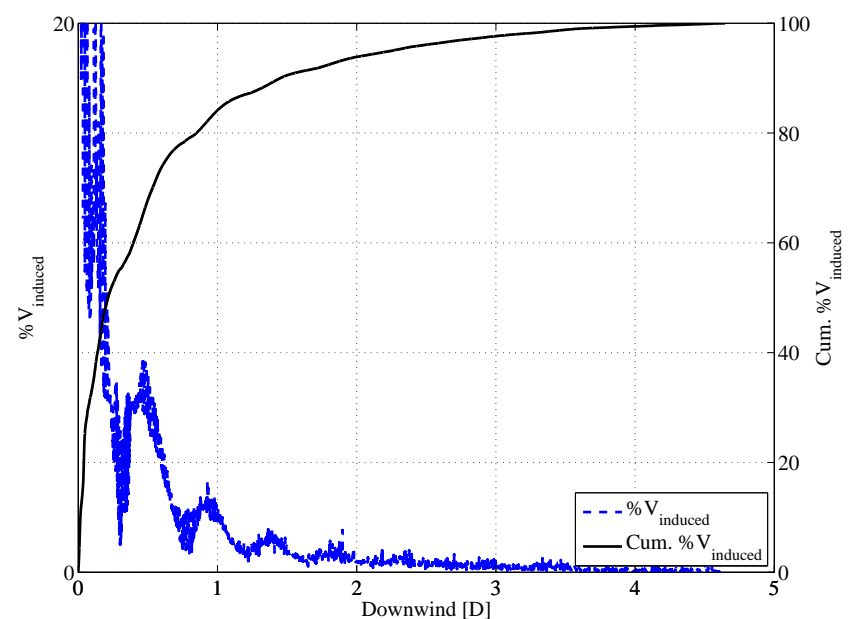

(a)

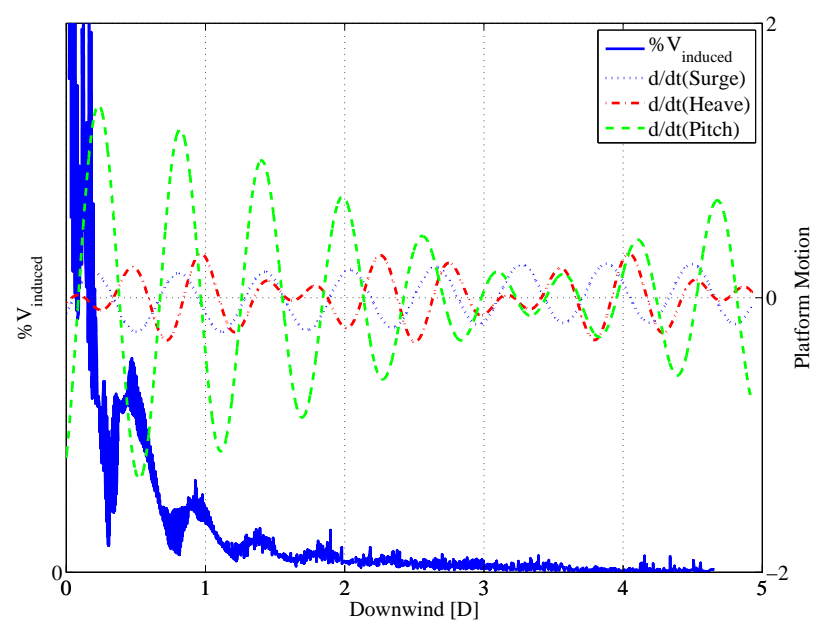

(b)

The accordion-like train of lobes illustrated in Figure 4 results in local peaks in induced velocity contributions in Figure 7. The associated advection wavelength may be computed in terms of rotor diameters $\left(\lambda_{D}\right)$ via Equation (6),

$$
\frac{U_{\infty}}{D f}=\lambda_{D}
$$

as a function of rotor diameter $(D)$, platform motion frequency $(f)$, and free stream velocity. For a free stream velocity of $6 \mathrm{~ms}^{-1}$ and an average pitch frequency of $f=0.08 \mathrm{~Hz}$ (Table 4), $\lambda_{D}=0.59 \mathrm{D}$. Figure 7 indicates that multiples of this wavelength correspond to peak negative slopes of the induced velocity as a function of downstream distance. This demonstrates that platform motions that occurred in the recent past, approximately $40 \mathrm{~s}$ for $2 \mathrm{D}$, can still influence the current loads on the rotor and that this is directly due to wake induction. Moreover, the positive or negative contributions to induced velocity correspond to the advection wavelength - the strength of the shed vorticity, and therefore induced velocity at the rotor, is linked to the platform motion. Virtually all of the velocity induced at the rotor is attributable to the first $4 \mathrm{D}$ of the wake, which is in agreement with Figure 6 . Also note that the pitch mode appears to be the primary driver of wake perturbations, as shown in Figure 7b. Peaks in platform pitch velocity are in phase with peaks in the induced velocity.

Figure 8 compares the Pareto plots for the OC3-Hywind spar-buoy under platform pitch and yaw both for below and above-rated wind speeds. As with Figure 7a, 100\% of the induced velocity is attributable to the first $5 \mathrm{D}$ of the wake; this appears to be independent of operating condition. Figure 8 a does not 
seem to demonstrate any induction at the rotor that is explicitly attributable to platform modes; there is no correlation between the pitch and yaw modes and any advection wavelength peaks in the $\% V_{\text {induced }}$ curve. Figure $8 \mathrm{~b}$ indicates that the above-rated pitch mode yields an advection wavelength of $4.9 \mathrm{D}$, close to the limit of non-negligible rotor induction.

Figure 8. Pareto plots of wake induced velocity at the lifting-line for the NREL-5MW turbine + OC3-Hywind spar-buoy under platform pitch and yaw. (a) Below-rated; (b) Above-rated.

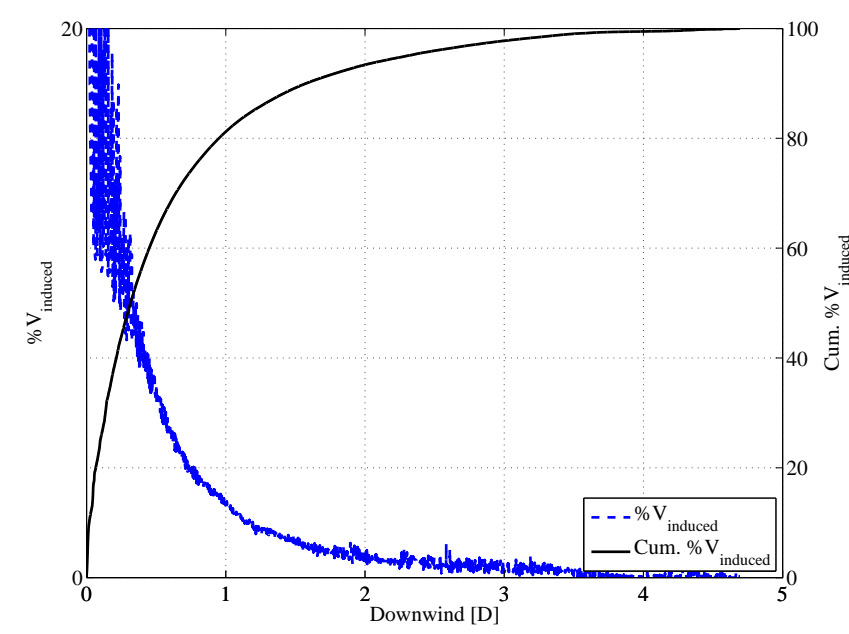

(a)

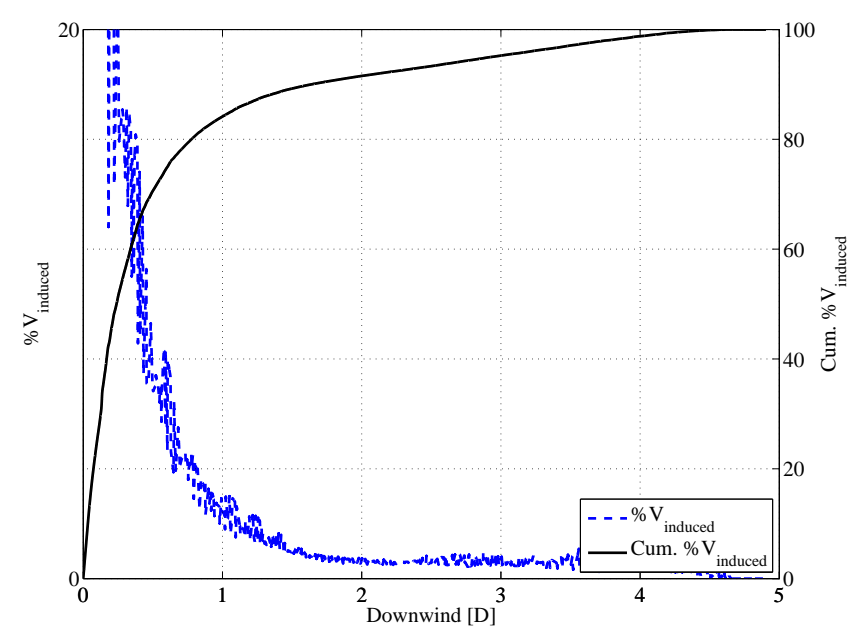

(b)

Table 5 outlines the results from all of the test cases, presenting the downstream distances for which $10 \%, 5 \%$, and $1 \%$ of the overall induced velocity at the rotor is attributable to the wake. The vorticity in the wake for below-rated cases at roughly $1 / 3 \mathrm{D}$ downstream translates into $10 \%$ of the overall induced velocity at the rotor. This increases to almost $0.5 \mathrm{D}$ downstream for above-rated cases. Note that the $1 \%$ induction distance values for the monopile cases are less than the below-rated yaw case, the rated barge pitch case, and the above-rated spar-buoy pitch case, indicating that platform motions affect the distance at which the wake induces loads on the rotor. This is due to the self-induction of the advected shed vorticity in the wake. The aforementioned accordion-like train of lobes or "bunches" of vorticity decelerate or accelerate the local flow field, thereby increasing or decreasing the influence of the shed vortices on induction at the rotor. Platform modes that increase the bunching of shed vorticity will increase the variability of induced velocity at the rotor, and by extension rotor loads.

Table 5. Downstream distance in rotor diameters of $1 \%, 5 \%$, and $10 \%$ overall wake-induced velocity at the rotor.

\begin{tabular}{|c|c|c|c|c|c|}
\hline$\underline{\text { Rotor Mode }}$ & Platform Type & Mode & $1 \% V_{\text {induced }}$ & $5 \% V_{\text {induced }}$ & $10 \% V_{\text {induced }}$ \\
\hline \multirow{4}{*}{ Below-rated } & Monopile & - & 4.33 & 0.64 & 0.33 \\
\hline & ITI Energy barge & Surge & 4.50 & 0.62 & 0.32 \\
\hline & ITI Energy barge & Heave & 4.34 & 0.65 & 0.33 \\
\hline & ITI Energy barge & Pitch & 1.87 & 0.58 & 0.20 \\
\hline
\end{tabular}


Table 5. Cont.

\begin{tabular}{|c|c|c|c|c|c|}
\hline Rotor Mode & Platform Type & Mode & $1 \% V_{\text {induced }}$ & $5 \% V_{\text {induced }}$ & $10 \% V_{\text {induced }}$ \\
\hline \multirow{5}{*}{ Below-rated } & ITI Energy barge & Surge + Heave + Pitch & 1.90 & 0.58 & 0.21 \\
\hline & OC3-Hywind spar-buoy & Pitch & 2.03 & 0.64 & 0.34 \\
\hline & OC3-Hywind spar-buoy & Yaw & 4.54 & 0.65 & 0.33 \\
\hline & OC3-Hywind spar-buoy & Pitch +Yaw & 2.61 & 0.64 & 0.34 \\
\hline & MIT/NREL TLP & Surge & 4.34 & 0.63 & 0.33 \\
\hline \multirow{2}{*}{ Rated } & Monopile & - & 2.02 & 0.79 & 0.43 \\
\hline & ITI Energy barge & Pitch & 2.20 & 0.76 & 0.48 \\
\hline \multirow{5}{*}{ Above-rated } & Monopile & - & 2.16 & 0.81 & 0.46 \\
\hline & ITI Energy barge & Pitch & 1.94 & 0.57 & 0.42 \\
\hline & OC3-Hywind spar-buoy & Pitch & 4.13 & 0.86 & 0.63 \\
\hline & OC3-Hywind spar-buoy & Yaw & 1.86 & 0.66 & 0.44 \\
\hline & OC3-Hywind spar-buoy & Pitch+Yaw & 3.56 & 0.69 & 0.45 \\
\hline
\end{tabular}

\section{Inflow Angles}

The wake-induced velocity at the rotor is what drives aerodynamic loads at the rotor and the overall performance of the turbine. This induced velocity will adjust the relative angle between the free stream flow and the blade sections, dynamically influencing the rotor loading. The angle of attack, $\alpha$, serves as a stand-in for the induction response of the rotor to wake influences and the possibility of stall. The sideslip (or yaw) angle, $\beta$, provides a sense of the validity of the obtained solutions and indicates instances of modeling challenges; LLT only takes into account flow parallel to the chord.

\subsection{Angle of Attack}

Figure 9 compares normalized span-varying $\alpha$ histograms of the monopile and the pitching barge and spar-buoy under below-rated loading. Normalizing the histograms at each spanwise location by the number of bin elements for each test case facilitates direct comparison between each of the test cases. The mean line as well as the first standard deviation $(1 \sigma)$ range are also plotted. Note that the $\alpha$ histograms are normally distributed for all of the cases, with the standard deviation varying inversely with span. 1P variations in monopile response are due to shaft tilt. Relative velocities inboard are low compared to those closer to the blade tip, resulting in a greater range of $\alpha$ values inboard. While the mean values are similar, the standard deviations shown for the barge in Figure $9 \mathrm{~b}$ are much greater than for either the monopile or the spar-buoy. This indicates that the pitching motion of the barge has a significant impact on the local flow field, greater than that observed for the monopile or spar-buoy, for below-rated operating conditions. The sensitivity of the local flow field to platform motions changes with operating condition, as demonstrated by Figure 10, which compares the normalized span-varying $\alpha$ histograms of the monopile and the pitching barge and spar-buoy under above-rated operating conditions. 
Figure 9. $\alpha$ histograms of the NREL-5MW turbine operating in below-rated conditions.

(a) Monopile; (b) Pitch, ITI Energy barge; (c) Pitch, OC3-Hywind spar-buoy.

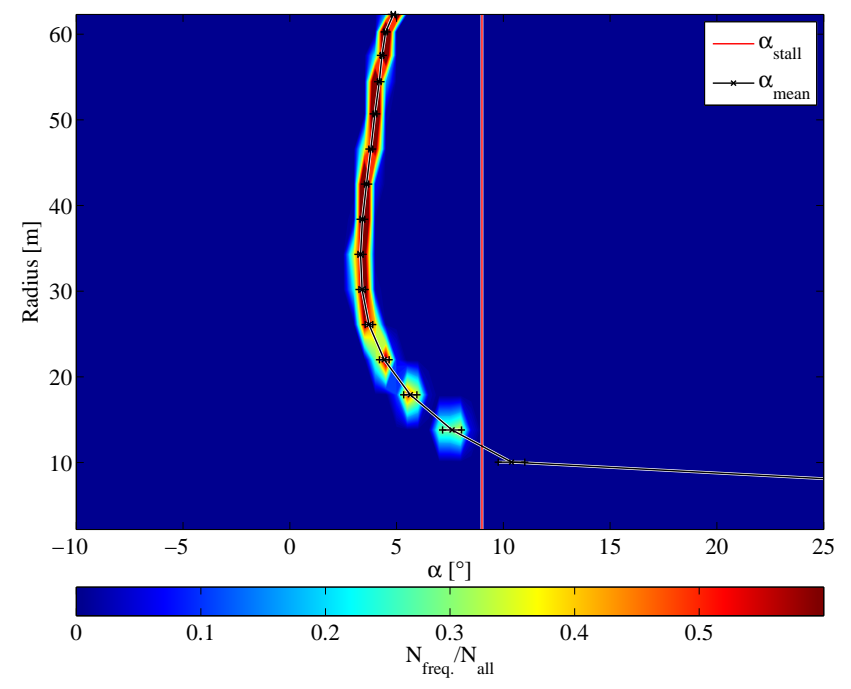

(a)

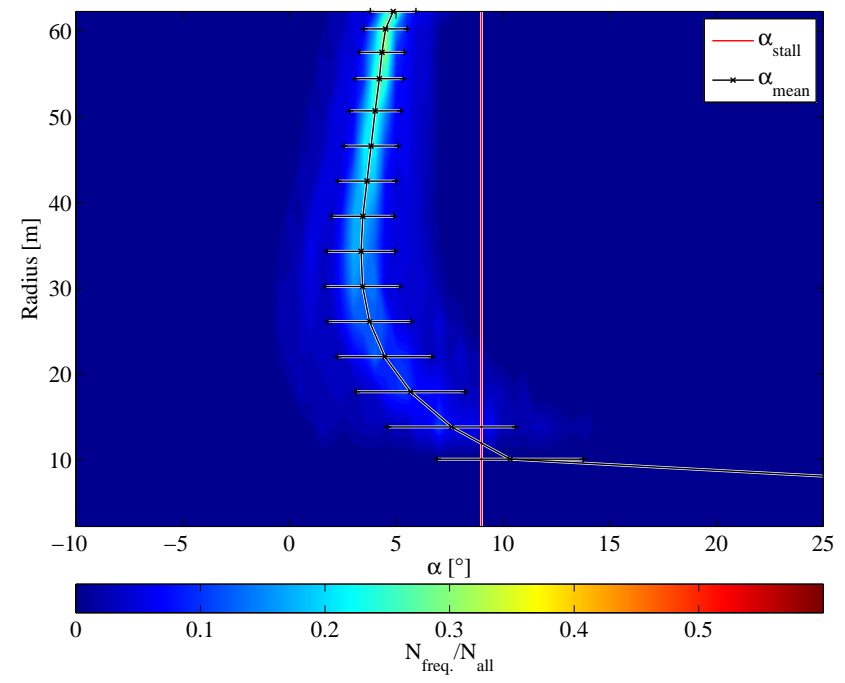

(b)

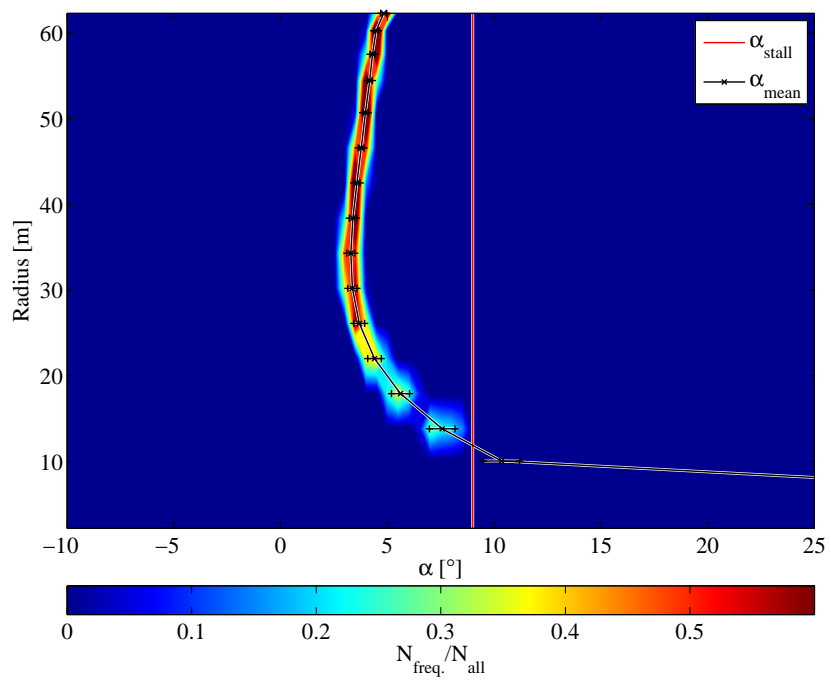

(c)

The mean spanwise $\alpha$ values are similar for each of the above-rated cases, but the standard deviations have changed. Both the barge (Figure 10b) and the spar-buoy (Figure 10c) have large standard deviations compared to the monopile, reflecting the significance of the platform motions for this load case. Note that for all of the cases presented in Figure 10, $\alpha$ becomes negative at the blade midspan.

Table 6 summarizes the mean $(\mu)$ value of $\alpha$ and $\sigma$ obtained from the histograms for the outboard ${ }^{2 / 3}$ of the blade for all of the test cases. The inboard $1 / 3$ is omitted in calculating these statistics due to the suspect nature of flow angle estimates near the root of a rotor blade [14]. 
Figure 10. $\alpha$ histograms of the NREL-5MW turbine operating in above-rated conditions.

(a) Monopile; (b) Pitch, ITI Energy barge; (c) Pitch, OC3-Hywind spar-buoy.

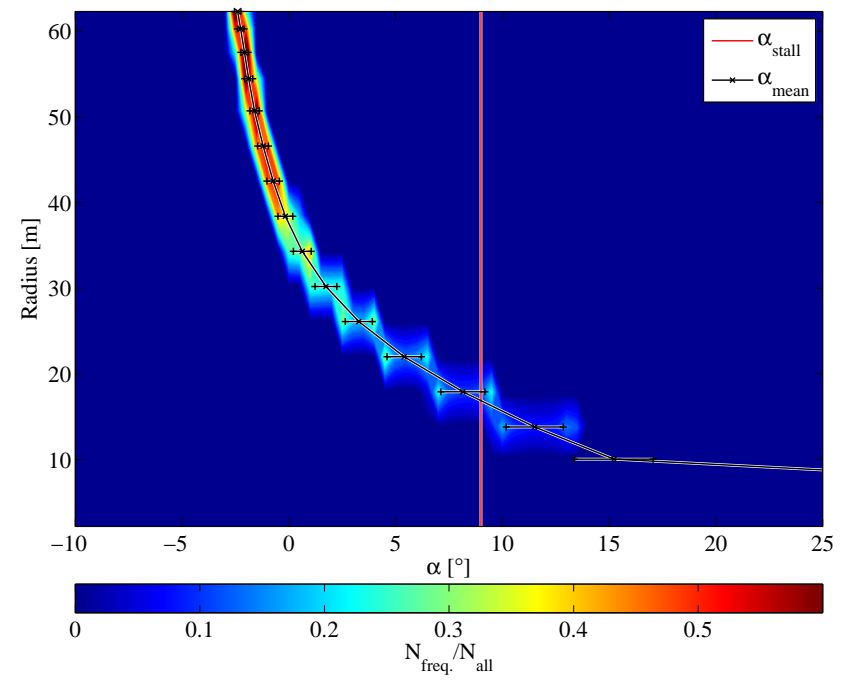

(a)

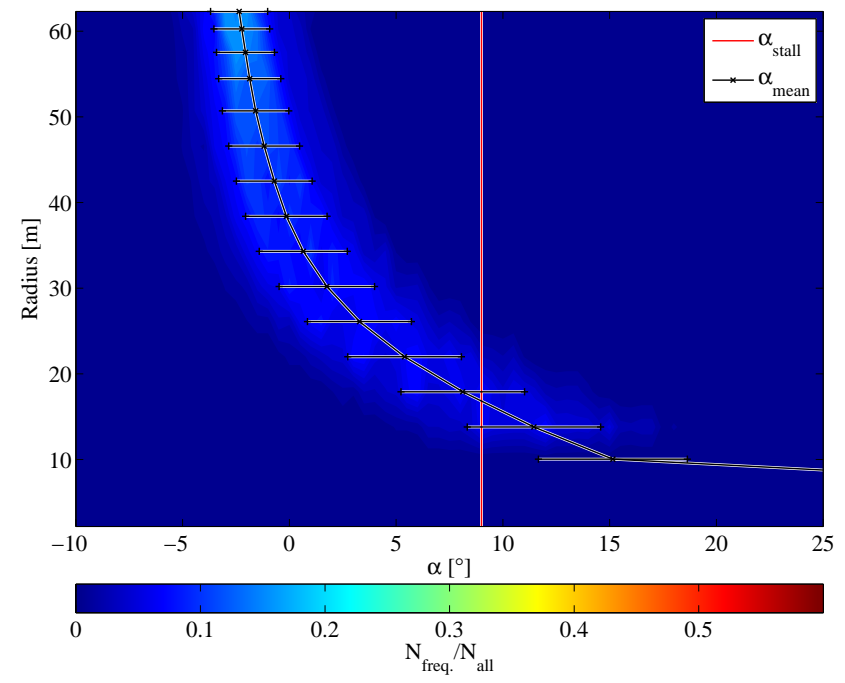

(b)

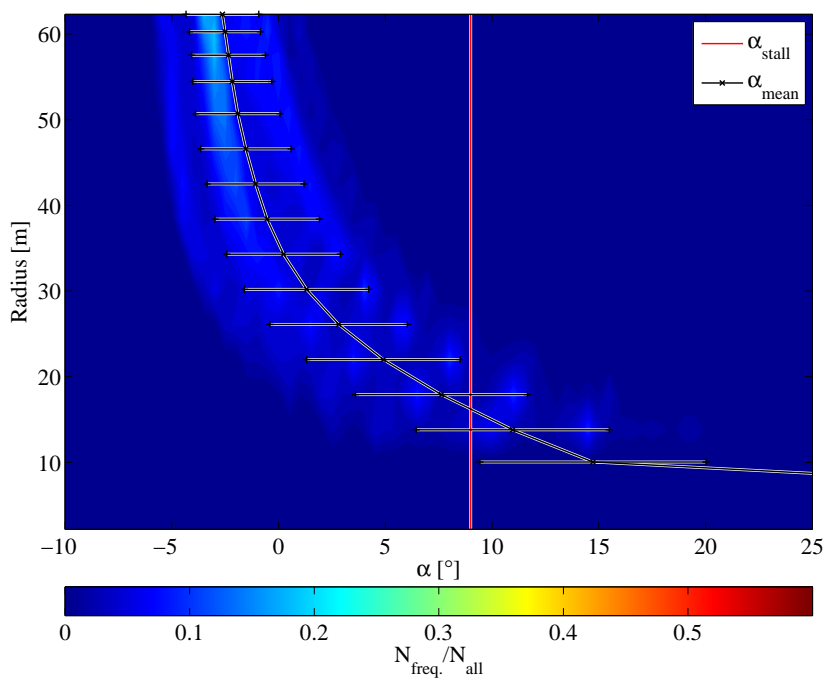

(c)

Table 6. Summary of mean and standard deviation values for outboard $2 / 3$ of the blade from $\alpha$ histogram results for NREL-5MW turbine.

\begin{tabular}{llcrr}
\hline Rotor Mode & Platform Type & Mode & $\boldsymbol{\mu}_{\boldsymbol{\alpha}}\left[^{\circ}\right]$ & $\boldsymbol{\sigma}_{\boldsymbol{\alpha}}\left[^{\circ}\right]$ \\
\hline & Monopile & - & 3.95 & 0.23 \\
& ITI Energy barge & Surge & 3.95 & 0.40 \\
Below-rated & ITI Energy barge & Heave & 3.95 & 0.24 \\
& ITI Energy barge & Pitch & 3.99 & 2.21 \\
& ITI Energy barge & Surge+Heave+Pitch & 4.03 & 3.59 \\
& OC3-Hywind spar-buoy & Pitch & 3.94 & 0.32 \\
& OC3-Hywind spar-buoy & Yaw & 3.95 & 0.23 \\
\hline
\end{tabular}


Table 6. Cont.

\begin{tabular}{llcrc}
\hline Rotor Mode & Platform Type & Mode & $\boldsymbol{\mu}_{\boldsymbol{\alpha}}\left[^{\circ}\right]$ & $\boldsymbol{\sigma}_{\boldsymbol{\alpha}}\left[^{\circ}\right]$ \\
\hline \multirow{2}{*}{ Below-rated } & OC3-Hywind spar-buoy & Pitch+Yaw & 3.90 & 0.27 \\
& MIT/NREL TLP & Surge & 3.95 & 0.27 \\
\hline \multirow{2}{*}{ Rated } & Monopile & - & 6.76 & 0.37 \\
& ITI Energy barge & Pitch & 6.78 & 1.67 \\
\hline \multirow{6}{*}{ Above-rated } & OC3-Hywind spar-buoy & Pitch & -0.10 & 0.80 \\
& Monopile & Yaw & -0.45 & 3.56 \\
& ITI Energy barge & Pitch & -0.53 & 0.96 \\
& OC3-Hywind spar-buoy & Pitch+Yaw & -1.03 & 3.12 \\
\hline & OC3-Hywind spar-buoy & &
\end{tabular}

It is clear from Table 6 that standard deviations for the pitching barge and spar-buoy under all loading conditions are greater than for the monopile, indicating that the pitching mode has a significant impact on the local flow field. Additionally, the yawing spar-buoy under above-rated loading also demonstrates a larger standard deviation.

Table 7 summarizes the percentage of time the $\alpha$ values exceeded the stall angle of attack, $9^{\circ}$ for the inboard $1 / 3$ (root), midspan $1 / 3$ (mid), and outboard 1/3 (tip) for the blade for all of the test cases. How to best model separation and the appropriate use of airfoil tables in modeling stall are questions of significant importance, though they are beyond the scope of this work. Identifying the percentage of time stall occurs, however, helps one gain a better sense of how often this may be a problem. Stall appears to be nonexistent for outboard blade sections and occurs more frequently inboard. For below-rated cases, the ITI Energy barge has the greatest frequency of inboard stall occurrence, while the spar-buoy and TLP appear to never stall. The barge stalls more frequently than the monopile for above-rated conditions, but the increase is modest. The larger stall frequencies associated with the monopile for rated and above-rated cases is attributable to the fixed blade pitch angle and lack of blade pitch control. Even so, stall appears to be of little concern for these test cases.

Table 7. Stall occurrence from $\alpha$ histogram results for NREL-5MW turbine.

\begin{tabular}{ccccc}
\hline Rotor Mode Platform Type & Mode & $\begin{array}{c}\boldsymbol{\alpha}_{\text {root }}>\boldsymbol{\alpha}_{\text {stall }} \\
{[\%]}\end{array}$ & $\boldsymbol{\alpha}_{\text {mid }}>\boldsymbol{\alpha}_{\text {stall }}$ & $\boldsymbol{\alpha}_{\text {tip }}>\boldsymbol{\alpha}_{\text {stall }}$ \\
{$[\%]$} & {$[\%]$} \\
\hline Monopile & - & 0.00 & 0.00 & 0.00 \\
ITI Energy barge & Surge & 0.29 & 0.00 & 0.00 \\
ITI Energy barge & Heave & 0.09 & 0.00 & 0.00 \\
Below-rated & Pitch & 3.58 & 0.00 & 0.00 \\
ITI Energy barge & Surge +Heave+Pitch & 6.46 & 0.00 & 0.00 \\
ITI Energy barge & Pitch & 0.08 & 0.00 & 0.00 \\
OC3-Hywind spar-buoy & Yaw & 0.00 & 0.00 & 0.00 \\
OC3-Hywind spar-buoy & & &
\end{tabular}


Table 7. Cont.

\begin{tabular}{|c|c|c|c|c|c|}
\hline Rotor Mode & Platform Type & Mode & $\begin{array}{c}\alpha_{\text {root }}>\alpha_{\text {stall }} \\
{[\%]}\end{array}$ & $\begin{array}{c}\alpha_{\text {mid }}>\alpha_{\text {stall }} \\
{[\%]}\end{array}$ & $\begin{array}{c}\alpha_{t i p}>\alpha_{\text {stall }} \\
{[\%]}\end{array}$ \\
\hline \multirow{2}{*}{ Below-rated } & OC3-Hywind spar-buoy & Pitch+Yaw & 0.00 & 0.00 & 0.00 \\
\hline & MIT/NREL TLP & Surge & 0.00 & 0.00 & 0.00 \\
\hline \multirow{2}{*}{ Rated } & Monopile & - & 17.49 & 0.00 & 0.00 \\
\hline & ITI Energy barge & Pitch & 16.48 & 2.39 & 0.16 \\
\hline \multirow{5}{*}{ Above-rated } & Monopile & - & 9.71 & 0.00 & 0.00 \\
\hline & ITI Energy barge & Pitch & 9.54 & 0.00 & 0.00 \\
\hline & OC3-Hywind spar-buoy & Pitch & 8.75 & 0.19 & 0.00 \\
\hline & OC3-Hywind spar-buoy & Yaw & 11.07 & 0.00 & 0.00 \\
\hline & OC3-Hywind spar-buoy & Pitch +Yaw & 7.31 & 0.00 & 0.00 \\
\hline
\end{tabular}

\subsection{Sideslip/Yaw Response}

The sideslip angle, $\beta$ is the angle between the local velocity vector and the chord line, projected onto the chord-blade plane. Lifting-line theory implicitly assumes this angle is zero; all of the flow is parallel to the chord. Figure 11 provides a sense of how often this occurs, comparing the normalized span-varying $\beta$ histograms of the monopile and the pitching barge and spar-buoy under below-rated operation. These histograms were normalized by the number of elements in all of the bins to facilitate direct comparison between each of the test cases and include the mean line as well as the $1 \sigma$-range.

Figure 11. $\beta$ histograms of the NREL-5MW turbine operating in below-rated conditions.

(a) Monopile; (b) Pitch, ITI Energy barge; (c) Pitch, OC3-Hywind spar-buoy.

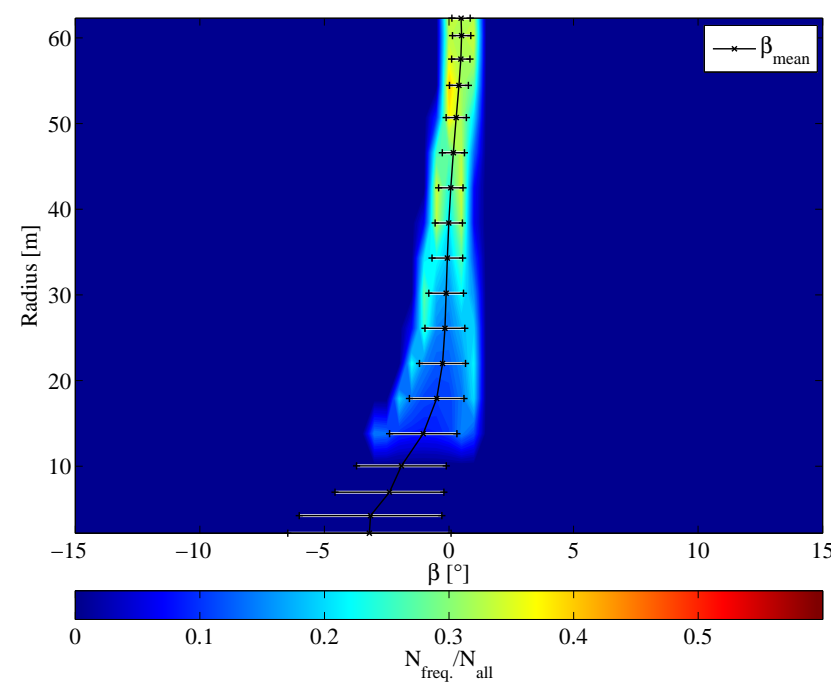

(a)

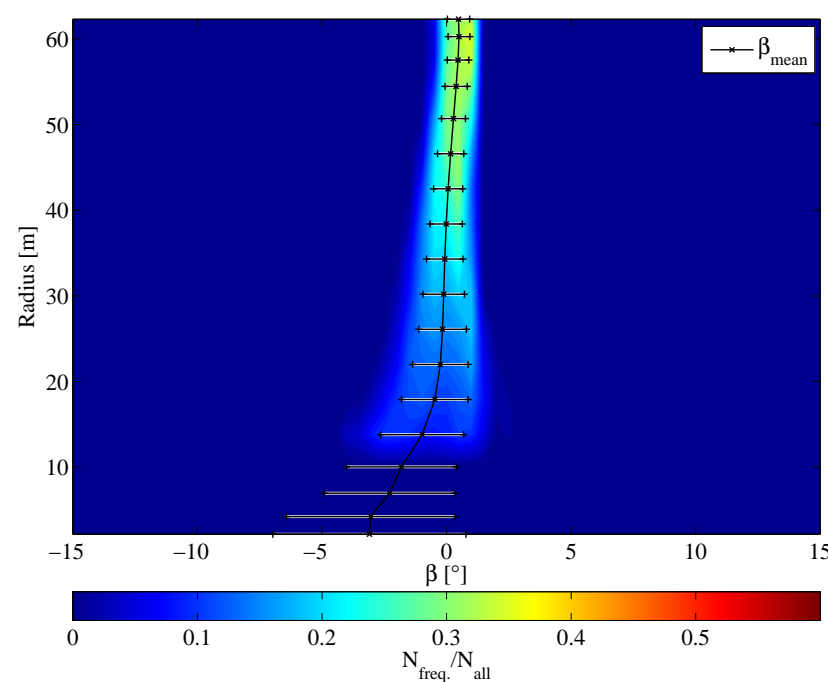

(b) 
Figure 11. Cont.

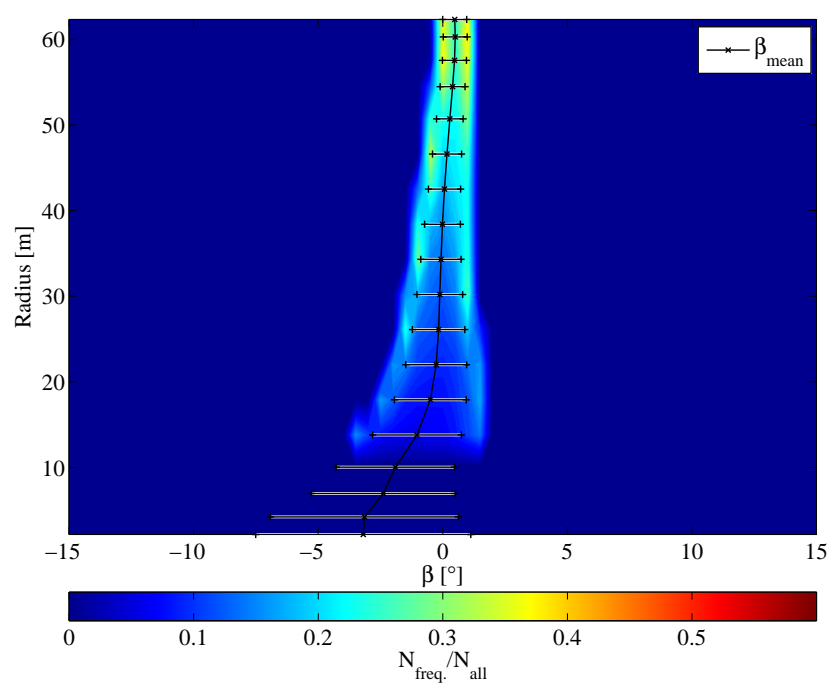

(c)

The sideslip angle is nominally zero and normally distributed for outboard blade sections. The $1 \sigma$-range for the floating platforms are comparable to the monopile, indicating that the $\beta$ distribution is due to the $1 \mathrm{P}$ variation in flow angle arising from the shaft tilt. The inboard $\beta$ shift demonstrated by the barge is due to the influence of the platform pitching motion and the magnitude of its effective velocity contribution relative to the free stream; as this velocity contribution becomes a smaller fraction of the overall local velocity for above-rated cases, the bimodal distribution creeps outboard. This is shown in Figure 12, which shows the histograms for the cases presented in Figure 11, but for above-rated conditions.

Figure 12. $\beta$ histograms of the NREL-5MW turbine operating in above-rated conditions.

(a) Monopile; (b) Pitch, ITI Energy barge; (c) Pitch, OC3-Hywind spar-buoy.

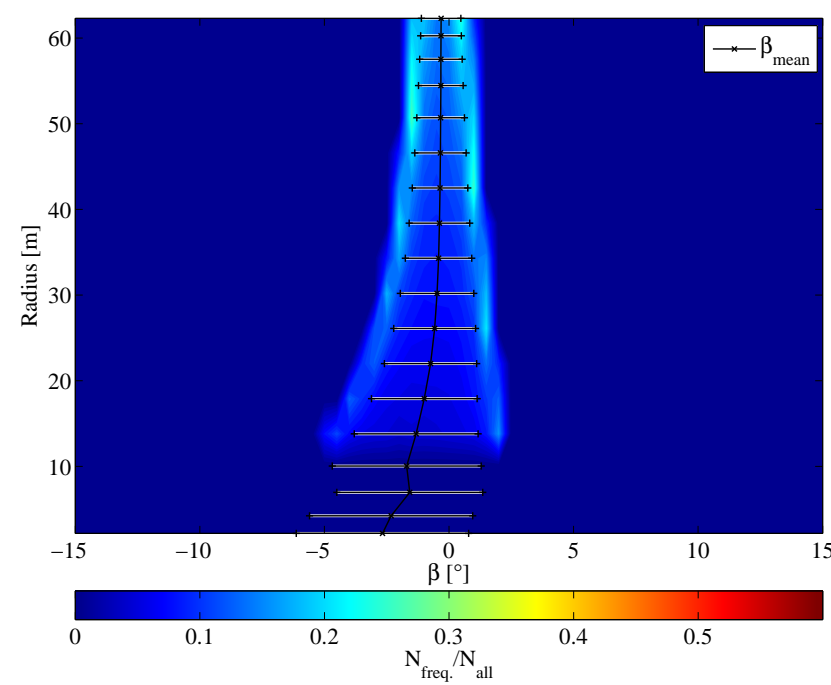

(a)

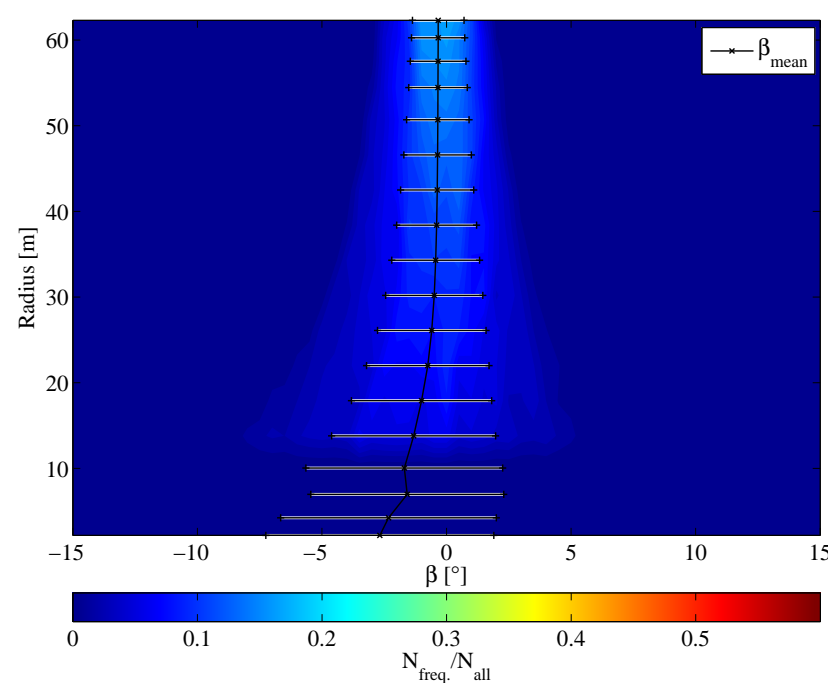

(b) 
Figure 12. Cont.

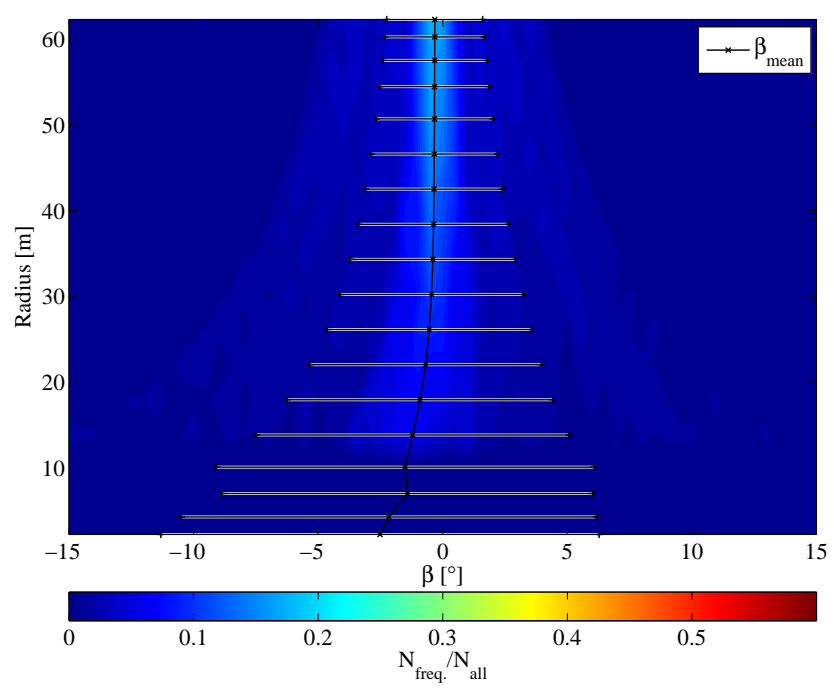

(c)

A significant increase in $\beta 1 \sigma$-range is shown by Figure 12 for both the monopile and floating cases. Note that the bimodal distribution has extended outboard for the monopile and barge cases, effectively increasing the variation, while the spar-buoy distribution appears to have expanded significantly.

Table 8 summaries the maximum $\beta$ histogram standard deviations and standard deviations obtained from the histograms for the outboard $2 / 3$ of the blade for all of the test cases. Note that all of the dynamic cases have greater variation in $\beta$ than the monopile cases, though the mean values are comparable within each group of operating conditions. The fraction of variation in the dynamic cases relative to the monopile cases suggests that a large amount of the variation in $\beta$, the bimodal response that was observed in Figures 11 and 12, is due to the 1P variation in flow direction.

Table 8. Summary of mean and standard deviation values for outboard $2 / 3$ of the blade from $\beta$ histogram results for NREL-5MW turbine.

\begin{tabular}{llcrr}
\hline Rotor Mode & Platform Type & Mode & $\boldsymbol{\mu}_{\boldsymbol{\beta}}\left[^{\circ}\right]$ & $\boldsymbol{\sigma}_{\boldsymbol{\beta}}\left[^{\circ}\right]$ \\
\hline & Monopile & - & 0.15 & 0.93 \\
& ITI Energy barge & Surge & 0.15 & 0.93 \\
& ITI Energy barge & Heave & 0.15 & 0.98 \\
& ITI Energy barge & Pitch & 0.15 & 1.11 \\
Below-rated & ITI Energy barge & Surge +Heave+Pitch & 0.14 & 1.19 \\
& OC3-Hywind spar-buoy & Pitch & 0.15 & 1.22 \\
& OC3-Hywind spar-buoy & Yaw & 0.15 & 0.93 \\
& OC3-Hywind spar-buoy & Pitch+Yaw & 0.15 & 1.22 \\
& MIT/NREL TLP & Surge & 0.15 & 0.93 \\
\hline \multirow{2}{*}{ Rated } & Monopile & - & 0.26 & 1.20 \\
& ITI Energy barge & Pitch & 0.25 & 1.66 \\
\hline
\end{tabular}


Table 8. Cont.

\begin{tabular}{llccc}
\hline Rotor Mode & Platform Type & Mode & $\boldsymbol{\mu}_{\boldsymbol{\beta}}\left[^{\circ}\right]$ & $\boldsymbol{\sigma}_{\boldsymbol{\beta}}\left[^{\circ}\right]$ \\
\hline \multirow{4}{*}{ Above-rated } & Monopile & - & -0.41 & 1.85 \\
& ITI Energy barge & Pitch & -0.42 & 2.46 \\
& OC3-Hywind spar-buoy & Pitch & -0.40 & 4.64 \\
& OC3-Hywind spar-buoy & Yaw & -0.58 & 2.13 \\
& OC3-Hywind spar-buoy & Pitch+Yaw & -0.40 & 4.76 \\
\hline
\end{tabular}

Table 9 summarizes the percentage of time the $\beta$ values exceeded $2.5^{\circ}$, violating the small spanwise angle assumptions used in lifting-line and momentum balance theories. Spanwise flow is minimal for below-rated and rated conditions, limited to the inboard $1 / 3$ of the blade and remaining within the small angle limits most of the time. This decreases substantially for above-rated cases; the small angle assumption is violated with greater frequency for decreasing tip speed ratios.

Table 9. Occurrence of large $\beta$ values from histogram results for NREL-5MW turbine.

\begin{tabular}{|c|c|c|c|c|c|}
\hline Rotor Mode & Platform Type & Mode & $\begin{array}{c}\beta_{\text {root }}>2.5^{\circ} \\
{[\%]}\end{array}$ & $\begin{array}{c}\beta_{\text {mid }}>2.5^{\circ} \\
{[\%]}\end{array}$ & $\begin{array}{c}\beta_{t i p}>2.5^{\circ} \\
{[\%]}\end{array}$ \\
\hline \multirow{9}{*}{ Below-rated } & Monopile & - & 2.02 & 0.00 & 0.00 \\
\hline & ITI Energy barge & Surge & 2.00 & 0.00 & 0.00 \\
\hline & ITI Energy barge & Heave & 2.18 & 0.00 & 0.00 \\
\hline & ITI Energy barge & Pitch & 3.10 & 0.04 & 0.00 \\
\hline & ITI Energy barge & Surge + Heave + Pitch & 3.51 & 0.25 & 0.00 \\
\hline & OC3-Hywind spar-buoy & Pitch & 3.63 & 0.00 & 0.00 \\
\hline & OC3-Hywind spar-buoy & Yaw & 1.98 & 0.00 & 0.00 \\
\hline & OC3-Hywind spar-buoy & Pitch + Yaw & 3.55 & 0.00 & 0.00 \\
\hline & MIT/NREL TLP & Surge & 1.97 & 0.00 & 0.00 \\
\hline \multirow{2}{*}{ Rated } & Monopile & - & 3.99 & 0.00 & 0.00 \\
\hline & ITI Energy barge & Pitch & 7.07 & 0.84 & 0.00 \\
\hline \multirow{5}{*}{ Above-rated } & Monopile & - & 7.69 & 3.59 & 0.00 \\
\hline & ITI Energy barge & Pitch & 10.22 & 8.59 & 2.79 \\
\hline & OC3-Hywind spar-buoy & Pitch & 12.00 & 14.78 & 13.02 \\
\hline & OC3-Hywind spar-buoy & Yaw & 9.68 & 7.25 & 0.43 \\
\hline & OC3-Hywind spar-buoy & Pitch +Yaw & 12.81 & 15.43 & 13.24 \\
\hline
\end{tabular}

\section{Unsteady Aerodynamics and Time Lag}

It is obvious that certain platform motions will have an impact on the local flow field of an OFWT, contributing to the effective local flow velocity. Less obvious is the temporal effect these platform motions may have on spanwise induction. As demonstrated by Sebastian and Lackner [1], platform 
motions may result in unsteady aerodynamics, observed as a lag in the loading response. Comparisons between the spanwise bound vorticity and the platform motions, normalized by maximum absolute value and offset to zero by the mean value, are presented in Figure 13 with respect to time.

Figure 13. Normalized spanwise bound vorticity for the NREL-5MW turbine. (a) Monopile, above-rated; (b) Surge, MIT/NREL TLP, below-rated; (c) Pitch+Yaw, OC3-Hywind spar-buoy, below-rated; (d) Pitch+Yaw, OC3-Hywind spar-buoy, above-rated; (e) Pitch, ITI Energy barge, above-rated.

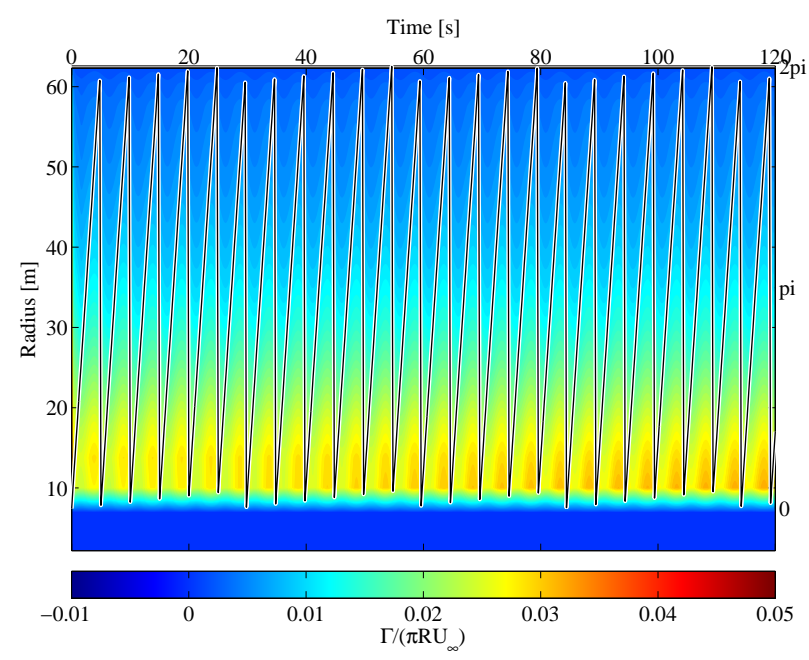

(a)

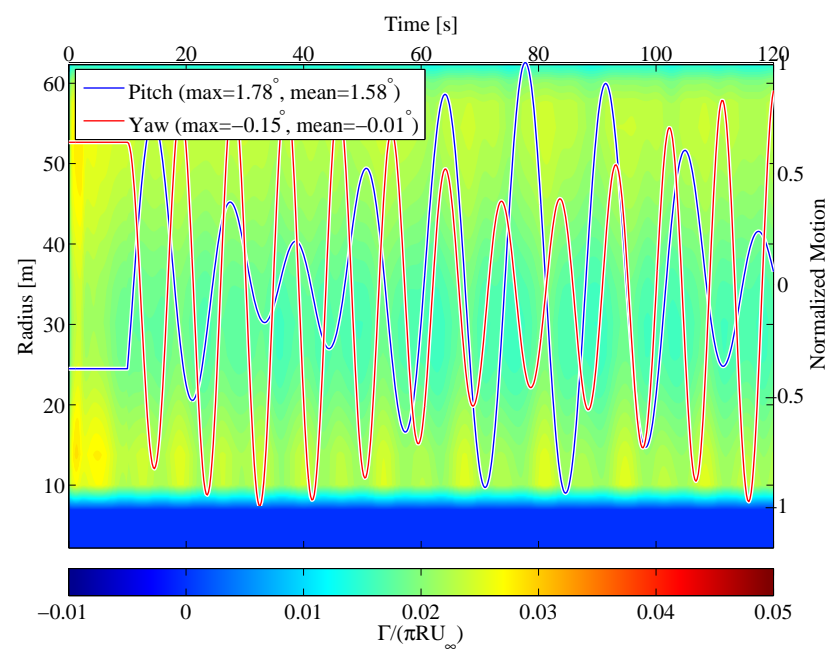

(c)

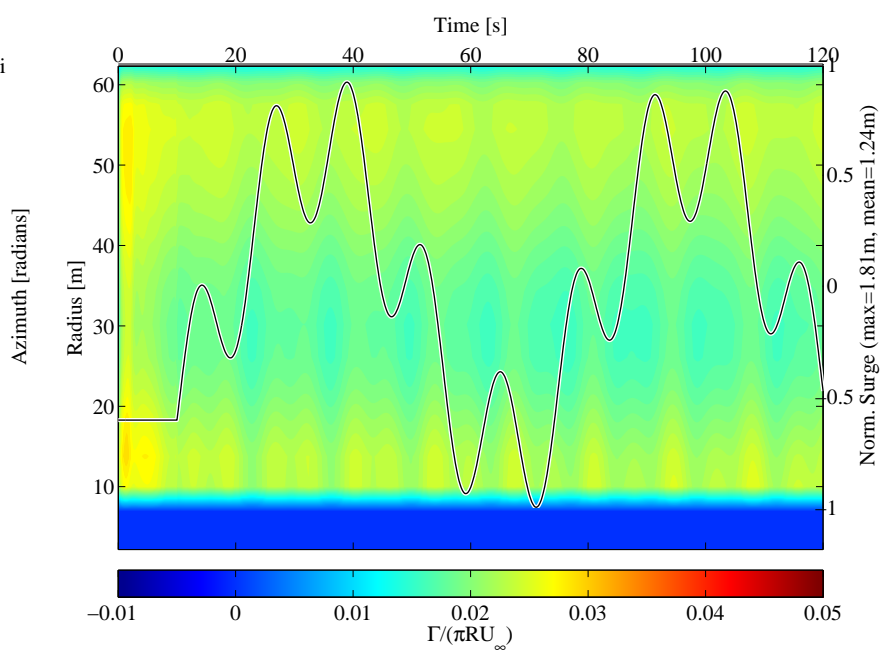

(b)

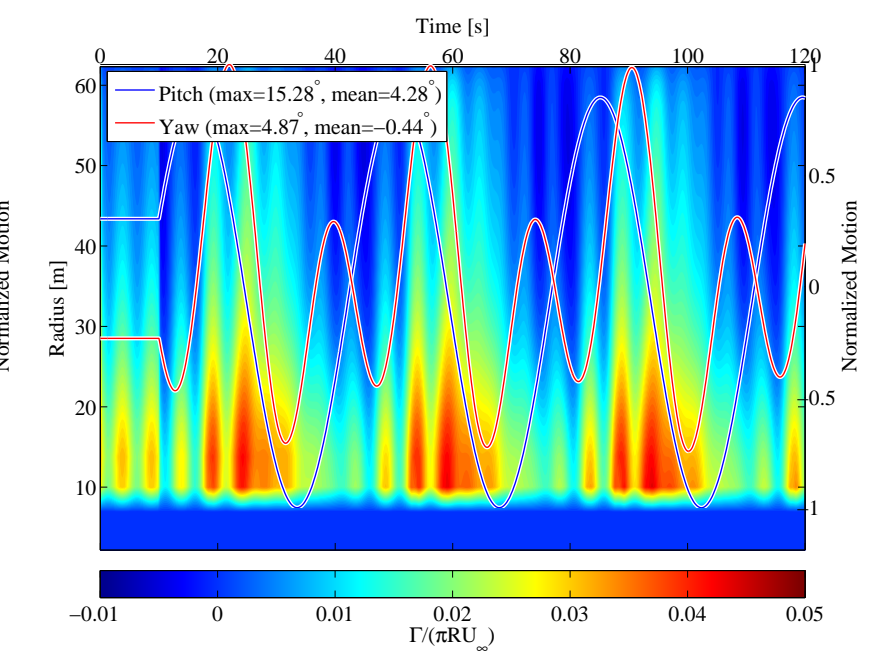

(d) 
Figure 13. Cont.

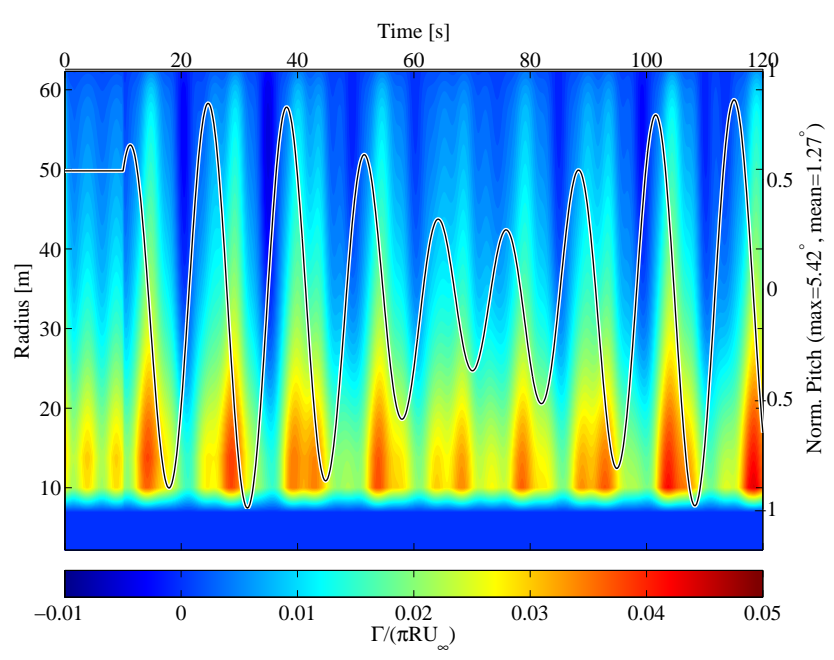

(e)

Figure 13a clearly shows the $1 \mathrm{P}$ influence on bound vorticity, which is also clearly present in the first $10 \mathrm{~s}$ of Figure 13d,e. The 1P influence continues to have a significant effect on the bound vorticity even after the platform motions begin. While windmill and propeller states are conventionally defined in terms of the axial induction factor, they may be defined in a more fundamental manner-if a rotor is imparting energy into the flow it is operating in a propeller or quasi-propeller state, and if it is extracting energy from the flow it is operating in a windmill state. Whether the rotor is imparting or extracting energy from the flow may be determined by observing the bound vorticity of the rotor blades. Figure 13c-e illustrate the influence of the platform pitching motions on the bound vorticity, with peaks (windmill state) and valleys (transitional to quasi-propeller state) trending with the pitch motion time derivative maxima and minima. The TLP in Figure 13b is most like the monopile in Figure 13a; the 1P influence is the only clear contributor to variation in bound vorticity.

The variation in bound vorticity due to platform pitching motions will lead to larger variations in loading on the rotor, thereby leading to increased fatigue. Also note that as the platform pitches back, negative bound circulation strengths occur, indicating transitions between windmill and quasi-propeller states.

\subsection{Spanwise Time Lag in Loading}

While it is clear that there is a lag between the platform motion and the resulting spanwise load, it is difficult to discern exactly how large this lag is along the span. A first-order loading response to changes in geometric angle of attack is expected; below a threshold frequency the flow is quasi-steady and as that threshold frequency is exceeded, the phase shift between the geometric angle of attack, $\alpha_{\text {geo }}$, (input) and the angle of attack, $\alpha$, (output) should increase [14]. Reduced frequency $(k)$, which falls out of the non-dimensionalized Navier-Stokes equations, is a dimensionless metric often used to characterize the degree of unsteadiness of an aerodynamic system due to a disturbance with some frequency, $\omega$. For 
$k>0.05$, the flow may be deemed unsteady [14]. This may be used to determine a disturbance threshold or demarcation frequency, $f_{d}$, above which the flow may be unsteady, as shown in Equation (7),

$$
f_{d}=\frac{0.05}{\pi} \frac{\sqrt{U_{\infty}^{2}+(r \Omega)^{2}}}{c}
$$

which is a function of chord length, $c$, at the local blade radius, $r$, rotor speed, $\Omega$, and free stream wind, $U_{\infty}$. We can assume a first-order low-pass filter to represent this lag in the loading, given by Equation (8).

$$
\frac{\text { output }}{\text { input }}=\frac{\alpha}{\alpha_{\text {geo }}}=\frac{K}{s \tau+1}
$$

where $K, s$, and $\tau$ are the gain, Laplace transform variable, and time constant of the first-order system, respectively.

Another approach involves the Carpenter and Friedovich dynamic inflow equation [15]. This equation may be rewritten in terms of the annular thrust coefficient, as shown in Equation (9)

$$
d C_{T}=4 a(1-a)+C_{R} \dot{a}
$$

where $C_{R}$ is a ratio of the annular size and the local velocity,

$$
C_{R}=\frac{16}{3 \pi U}\left(\frac{R_{2}^{3}-R_{1}^{3}}{R_{2}^{2}-R_{1}^{2}}\right)
$$

We can calculate the Laplace transform of Equation (9) to obtain a transfer function that reflects the unsteady aerodynamics modeled by the Carpenter and Friedovich dynamic inflow equation. Note that this equation is nonlinear; it must be linearized before it can be transformed. The term $4 a^{2}$ may be expanded via Taylor series, yielding Equation (11),

$$
d C_{T} \simeq 4 a-\left[4 a_{0}^{2}+8 a_{0}\left(a-a_{0}\right)\right]+C_{R} \dot{a}
$$

where $a_{0}$ is the point about which the Taylor series is linearized. Assuming a step change in $d C_{T}$ yields Equation (12), which is the second-order Laplace transform of Equation (11).

$$
\frac{\text { output }}{\text { input }}=\frac{a}{d C_{T}}=\frac{s-4 a_{0}^{2}}{C_{R} s^{2}+s\left(4-8 a_{0}\right)}
$$

Note that if $a_{0}$ is zero, Equation (11) reduces to Equation (8). The time constant of the resulting first-order transfer function is equal to $C_{R} / 4$, where the gain is $K=1$. This was the same result obtained by Øye [16] and Leishman [14].

Because the frequencies of the geometric and induced $\alpha$ should be similar, a non-linear least squares fitting approach is used to compute the time constant $\tau$ along the span for each of the test cases. Figures 14-16 show how $\tau$ varies with span for each of the platform configurations and load cases, with $C_{R} / 4$ included for comparison (black curves, with solid, dashed, and dot-dashed lines corresponding to below-rated, rated, and above-rated conditions). 
Figure 14. Span-varying $\alpha$ time constants of the NREL-5MW turbine with monopile and MIT/NREL TLP.

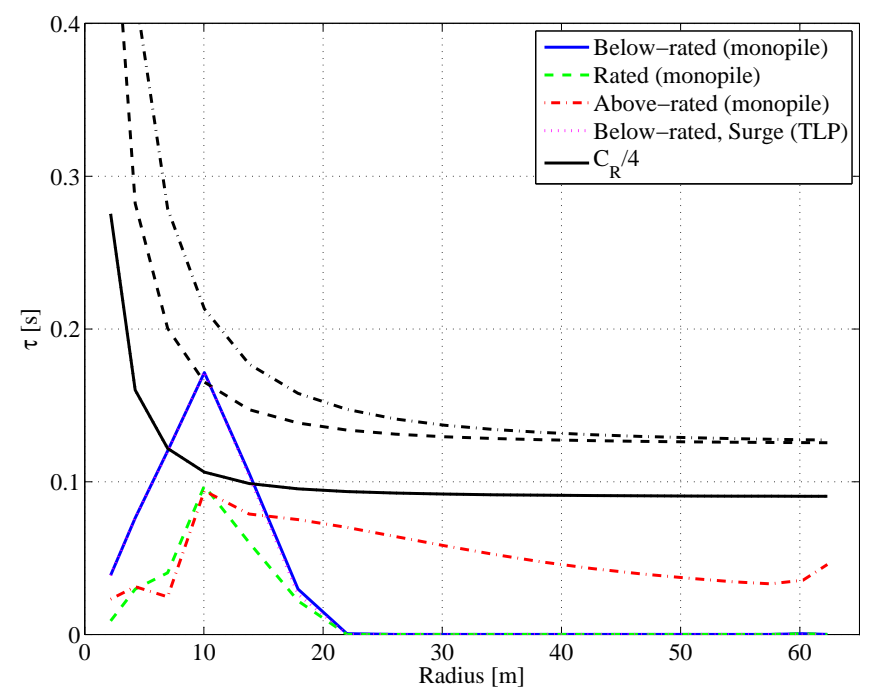

Figure 15. Span-varying $\alpha$ time constants of the NREL-5MW turbine + ITI Energy barge.

(a) Below-rated; (b) Platform pitching.

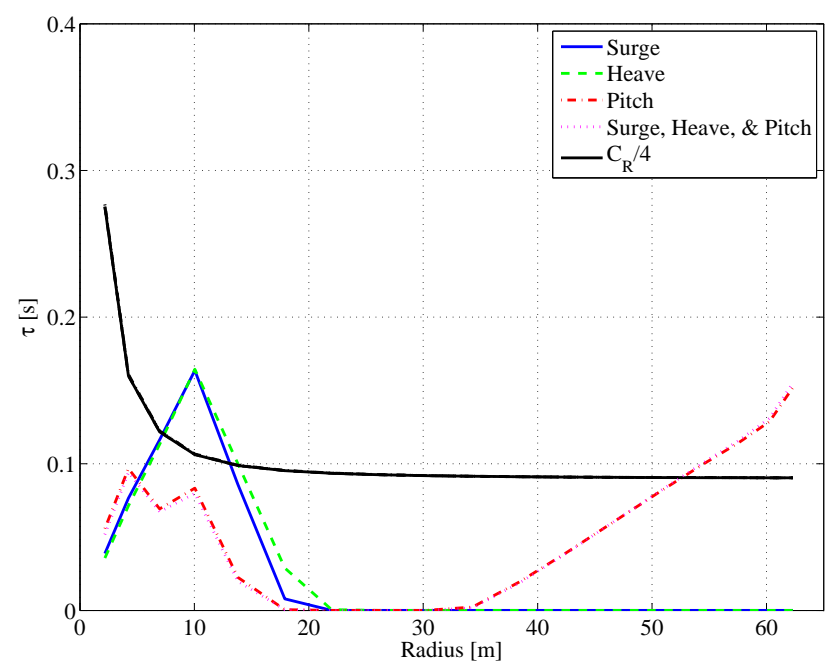

(a)

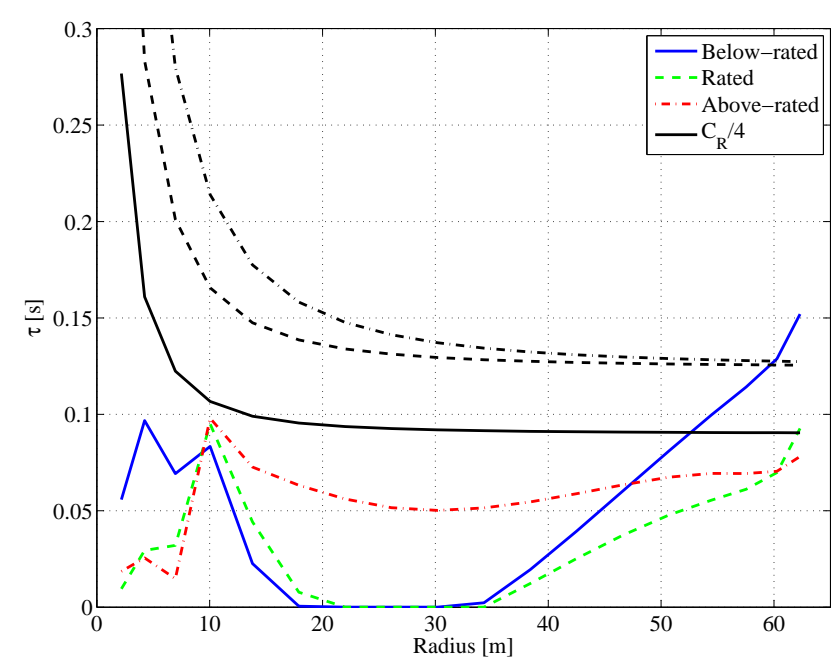

(b)

Figure 14 compares the estimated time constants for the monopile and the TLP. Note that the $\tau$ profile of the surging TLP under below-rated loading is similar to the monopile under rated conditions; throughout this study, the TLP has demonstrated characteristics similar to that of the monopile. Also note the spanwise minimum that occurs around the midspan of the blade for the monopile under below-rated conditions. Recall the $\beta$ histograms (Figure 12) and the bound vorticity plots (Figure 13); bimodal distributions in $\beta$ and spanwise transitions to low or negative bound circulation values occurred around the midspan as well. 
Figure 16. Span-varying $\alpha$ time constants of the NREL-5MW turbine + OC3-Hywind spar-buoy. (a) Below-rated; (b) Above-rated.

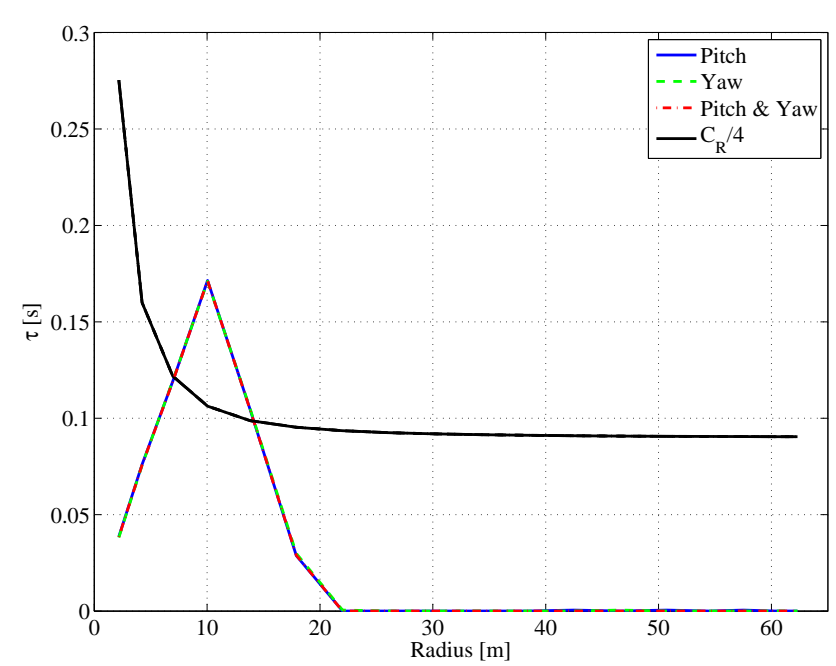

(a)

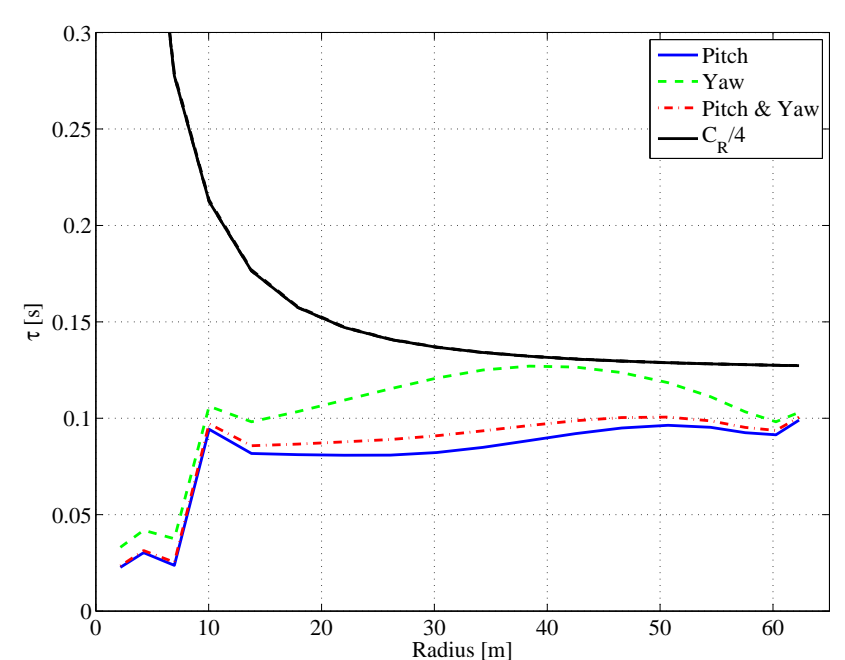

(b)

Figure 15, which shows the estimated time constants for the barge, demonstrates a slightly different response. Figure 15a shows that surge and heave modes have similar spanwise $\tau$ profiles to the TLP in Figure 14. The pitch mode, however, also has a similar $\tau$ distribution to the multiple-DOF case, indicating that the barge pitch mode is the aerodynamically-significant mode that causes the barge aerodynamic response to differ from the monopile. Comparing the barge pitching modes for all three load cases in Figure $15 \mathrm{~b}$ shows that the $\tau$ value is nominally $0.05 \mathrm{~s}$. Figure 16 indicates that the time constant for the spar-buoy is heavily influenced by the operating condition of the rotor, with $\tau$ dropping to zero for most of the span for below-rated conditions and nominally $0.1 \mathrm{~s}$ for above-rated conditions.

Recall that time lag is due to induction at the rotor, which is due to the wake. Figures 4 and 5 show (via opacity of the wake) that as the tip speed ratio increases, the induction at the midspan is lower than at the root and tip of the blade. This explains the prominent spanwise minimum in $\tau$ for below-rated conditions; the strength of the root and tip vortices are such that induction, and therefore lag, should be largest at these locations. Additionally, the ITI Energy barge with platform pitching appears to have sufficient induction across the span such that this $\tau$ minimum is not as prominent for above-rated conditions. The free stream wind convects the vortices downstream such that the relative influence of the tip vortices is minimized. This is also the case for the spar-buoy in above-rated operating conditions. It may be that the nonlinear relationship between $a$ and $\dot{a}$ given by Equation (9) invalidates the assumptions made in reformulating the equation as a first-order linear transfer function. The nonlinearity of this relationship is expected to increase with tip speed ratio (i.e., decreasing wind speed). $\tau$ should approach $C_{R} / 4$ with increasing wind speed, and this appears to agree with the observed sensitivity of the $\tau$ distributions shown in Figures 14 and 16b. 


\subsection{Time Lag Effects on Rotor Performance}

The relationship between the geometric and induced angles of attack for a wind turbine rotor varies along the span. Rotor-scale performance metrics like thrust, torque and power are the summation of all of the elemental spanwise contributions. Such outputs may serve as a system response to platform motions; a system identification may be used to characterize the relationship.

Figure 17 shows the thrust response of the OC3-Hywind spar-buoy, compared to the platform pitch and yaw time series for below and above-rated operating conditions. Immediately evident is the minimal impact platform yaw has on rotor thrust calculations, particularly for below-rated conditions. Platform pitch, on the other hand, yields an observable and significant response in rotor thrust. This may be explained by considering the effective wind shear across the rotor due to the different platform motions. A rotor on a pitching platform will experience an additional positive or negative wind shear corresponding to a windward or leeward pitch, respectively. A rotor on a yawing platform, however, will experience a positive wind shear on half of the rotor and a negative wind shear on the other half. As a result, most of the influence of platform yaw on the overall rotor cancels out.

Figure 17. Thrust response of the NREL-5MW turbine + OC3-Hywind spar-buoy. (a) Pitch, below-rated; (b) Yaw, below-rated; (c) Pitch, above-rated; (d) Yaw, above-rated.

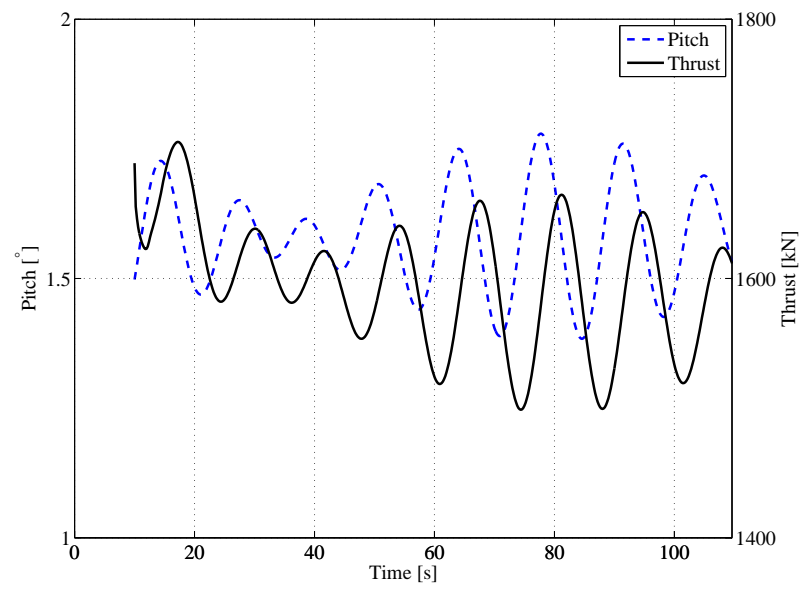

(a)

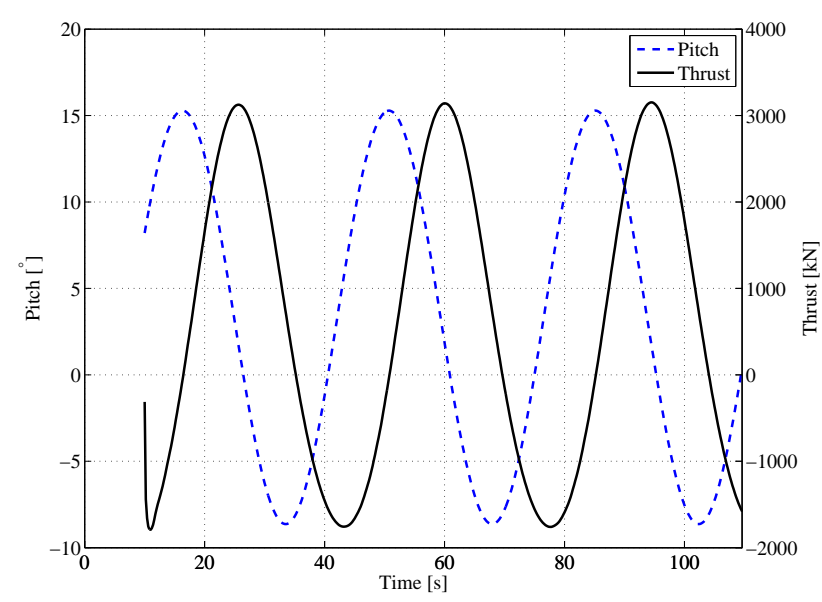

(c)

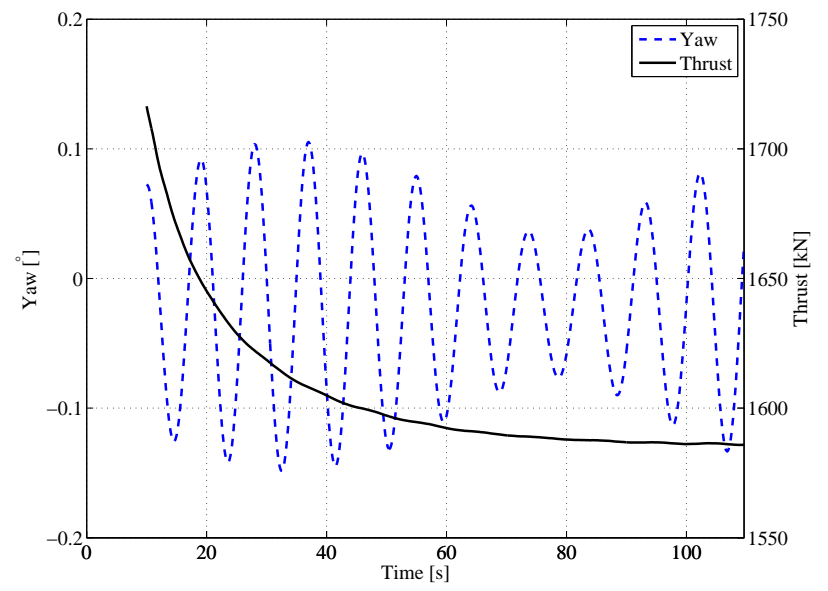

(b)

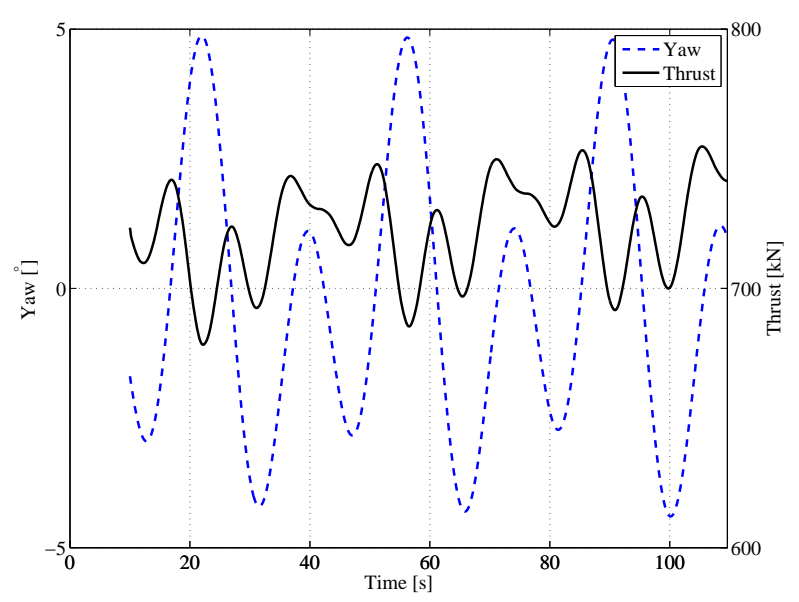

(d) 
Figure 18 compares the multiple-DOF cases, showing how rotor thrust compares to the normalized platform motions. Again, platform pitch appears to drive the thrust response. Note that, as with Figure 17, the thrust time series is approximately $90^{\circ}$ out-of-phase with the platform pitch motion. It is the time derivative of the platform motion, the effective velocity contribution, that dictates the delay and overall variation in rotor thrust response.

Figure 18. Thrust response of the NREL-5MW turbine under multiple-DOF. (a) ITI Energy barge, below-rated; (b) OC3-Hywind spar-buoy, below-rated; (c) OC3-Hywind spar-buoy, above-rated.

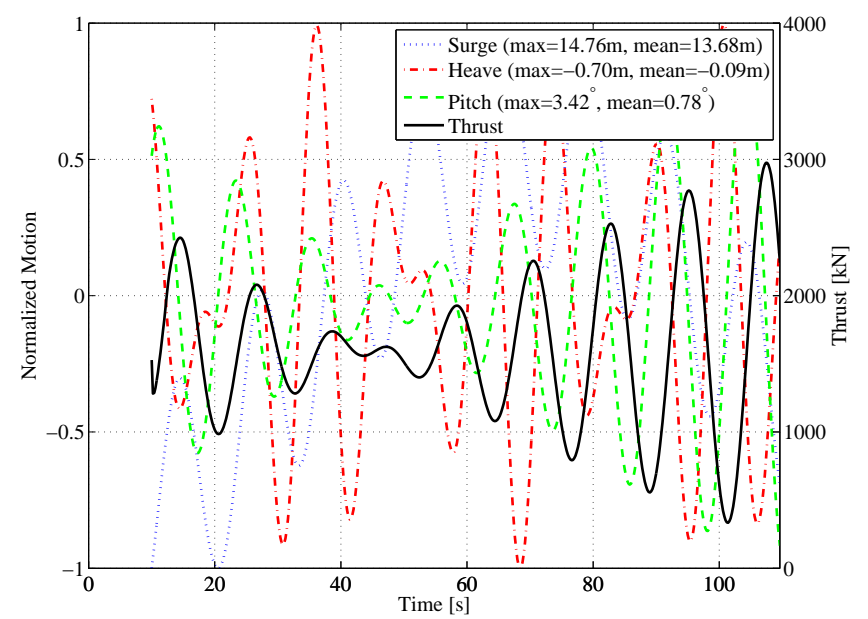

(a)

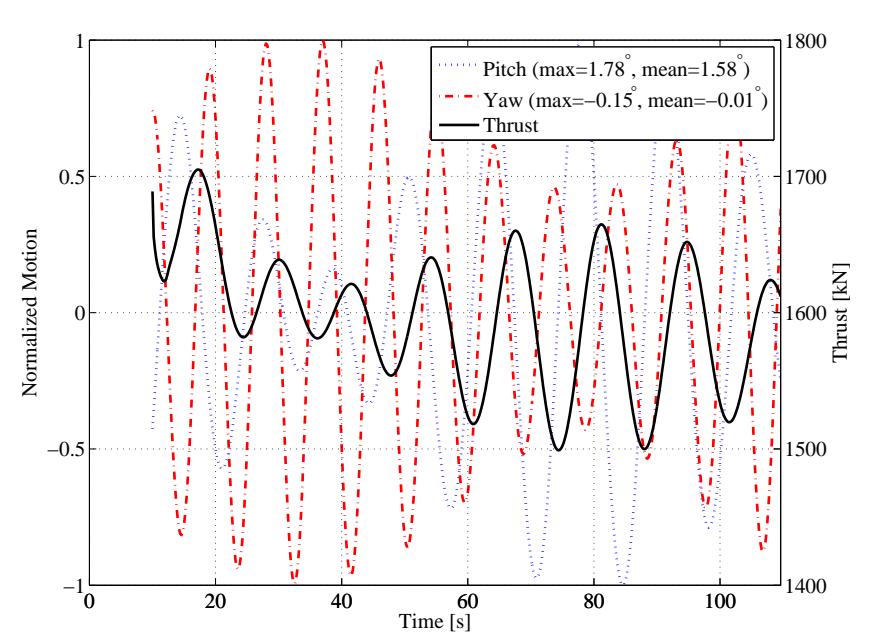

(b)

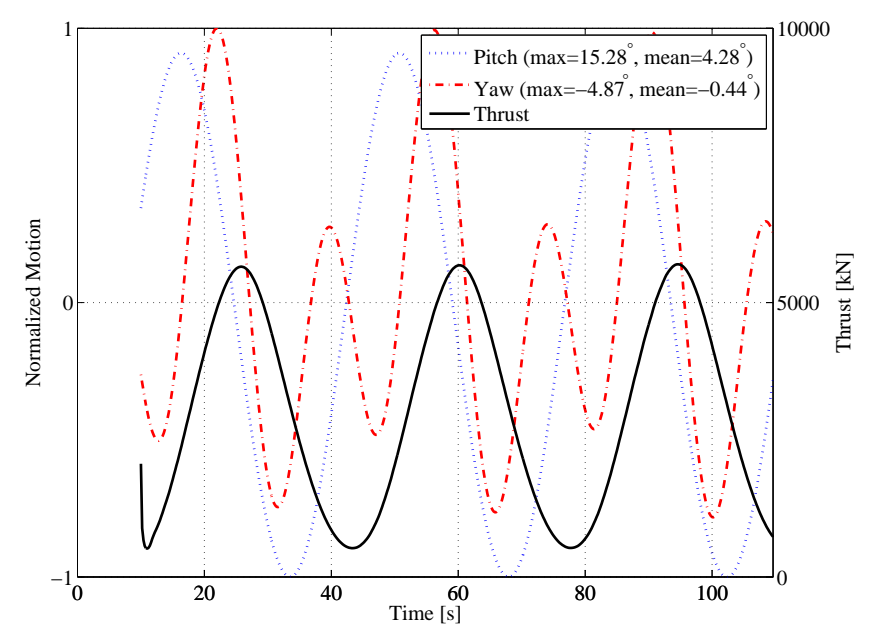

(c)

The peak platform motion-induced velocities may be directly compared to the peak thrust estimates, yielding an estimate for the time lag. Figure 19 shows this comparison for the multiple-DOF cases; lag is clearly evident. Additionally, it appears to be attributable almost entirely to the pitch mode. 
Figure 19. Overlay of peak motion-induced velocities to peak thrust values for the NREL-5MW turbine under multiple-DOF. (a) ITI Energy barge, below-rated; (b) OC3-Hywind spar-buoy, below-rated; (c) OC3-Hywind spar-buoy, above-rated.

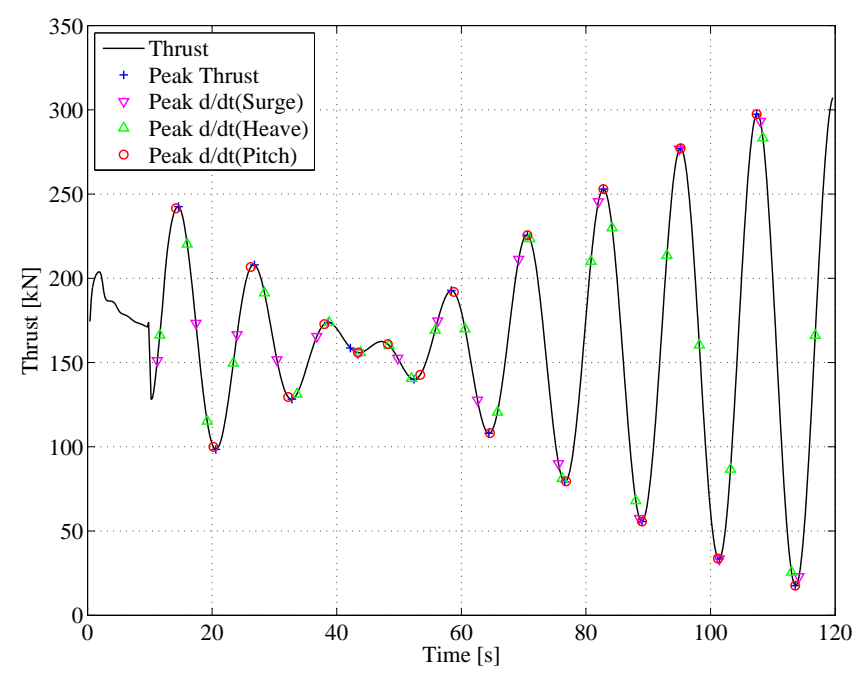

(a)

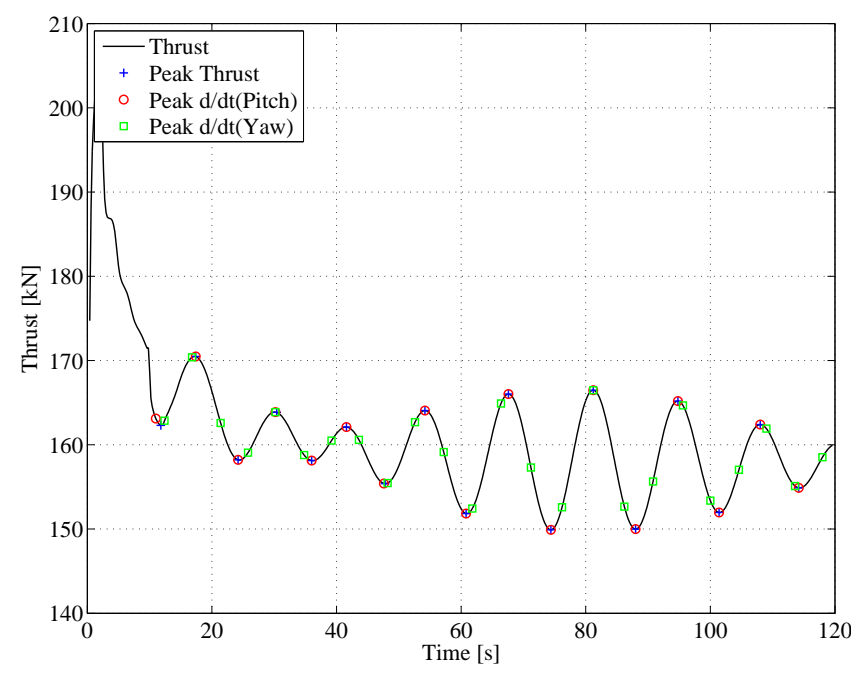

(b)

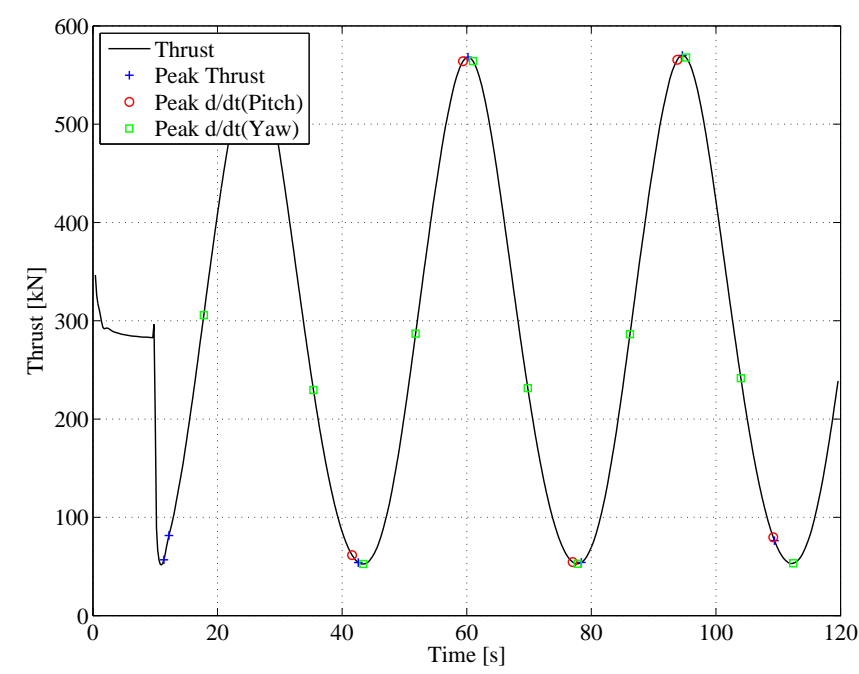

(c)

\section{Propeller State Transition Occurrence}

Propeller state transitions may occur with greater frequency for OFWTs. WInDS computes the axial induction factor such that positive values indicate windmill states and negative values indicate propeller states [12]. Table 10 shows the percentage of time during each simulation that each test case was operating in the propeller state. 
Table 10. Percentage of time operating in propeller state for NREL-5MW turbine.

\begin{tabular}{llcccc}
\hline \multirow{2}{*}{ Rotor Mode } & Platform Type & Mode & $\begin{array}{c}\boldsymbol{a}_{\text {root }}<\mathbf{0} \\
{[\mathbf{\%}]}\end{array}$ & $\begin{array}{c}\boldsymbol{a}_{\text {mid }}<\mathbf{0} \\
{[\%]}\end{array}$ & $\begin{array}{c}\boldsymbol{a}_{\text {tip }}<\mathbf{0} \\
{[\mathbf{\%}]}\end{array}$ \\
\hline \multirow{6}{*}{ Below-rated } & & 42.83 & 0.00 & 0.00 \\
& Monopile & - & 42.83 & 0.00 & 0.00 \\
& ITI Energy barge & Surge & 42.83 & 0.00 & 0.00 \\
& ITI Energy barge & Heave & 36.67 & 0.00 & 0.00 \\
& ITI Energy barge & Pitch & 36.07 & 0.00 & 0.00 \\
& ITI Energy barge & Surge+Heave+Pitch & 42.83 & 0.00 & 0.00 \\
& OC3-Hywind spar-buoy & Pitch & 42.83 & 0.00 & 0.00 \\
& OC3-Hywind spar-buoy & Yaw & 42.83 & 0.00 & 0.00 \\
& OC3-Hywind spar-buoy & Pitch+Yaw & 42.83 & 0.00 & 0.00 \\
\hline \multirow{3}{*}{ Rated } & MIT/NREL TLP & Surge & 42.83 & 0.00 & 0.00 \\
& Monopile & - & 42.83 & 0.00 & 0.00 \\
\hline & ITI Energy barge & Pitch & 42.88 & 0.53 & 0.73 \\
& Monopile & - & 41.38 & 0.69 & 9.90 \\
& ITI Energy barge & Pitch & 36.31 & 6.28 & 23.93 \\
Above-rated & OC3-Hywind spar-buoy & Pitch & 41.56 & 26.44 & 98.35 \\
& OC3-Hywind spar-buoy & Yaw & 35.67 & 7.24 & 27.07 \\
\hline & OC3-Hywind spar-buoy & Pitch+Yaw & & &
\end{tabular}

The monopile appears to have an unusually large propeller state occurrence frequency; it has a greater frequency than all of the dynamic cases for near the root. Note that the propeller state occurrence frequency is generally greatest closest to the root and tip for above-rated conditions. This indicates that the tip and root vortices have a significant influence on propeller state occurrence; this is not modeled using momentum balance approaches in a physically consistent manner. Most importantly, the propeller state occurrence frequency increases for the outboard blade sections of the floating systems relative to the monopile. This indicates that platform motions have a significant impact on propeller state occurrence, particularly at low tip speed ratios, which is in agreement with earlier results [1].

\section{Discussion}

The test cases outlined in this chapter were selected to cover a wide range of turbine design and operating conditions while minimizing the number of required simulations. The simulated wake is more unstable for below-rated cases than above-rated cases. Alternatively, wake stability is inversely related to tip speed ratio, and this is due to the rate of vortex filament convection and the prominent wake self-induction that takes place at high tip speed ratios. Also, roll-up of the wake at the roots and tips of the blades results in greater induction at the rotor, and this is amplified for high tip speed ratios. In terms of wake stability, the platform pitching mode appears to have the most impact, both for the ITI Energy barge and the OC3-Hywind spar-buoy.

Induction at the rotor appears to be influenced by the platform pitching mode via bunches of shed vorticity that have convected downstream. This memory effect of previous platform motions on the 
current induction at the rotor is significant, although its influence, and the influence from all of the simulated wakes, drops below $1 \%$ of overall induced velocity at the rotor around $2 \mathrm{D}$ downstream, and nearly $100 \%$ of the induced velocity at the rotor appears to be attributable to the wake within 4D.

Analyses of the flight angles, $\alpha$ and $\beta$, continue to demonstrate that platform pitching is the primary mode of interest. It has a significant impact on the standard deviations of $\alpha$ and increases the likelihood of stall. The yaw mode also appears to have some impact compared to the monopile, but only for above-rated conditions. The sideslip angle demonstrates a normal distribution for outboard blade sections that turns into a bimodal distribution for inboard sections. This shift in $\beta$ response is due to the platform pitching motion and its effective velocity contribution relative to the free stream. The bimodal distribution creeps outboard as this velocity contribution becomes a smaller fraction of the overall local velocity. Additionally, this shift appears to correspond to the drop in spanwise induction observed in the simulated wakes.

The spanwise bound vorticity is heavily influenced by the platform motions, with peaks in vorticity matching up with peaks in platform-induced velocities. Also observed are negative bound vorticity values, located on outboard blade sections. These negative values match up with leeward platform pitching motions and indicate transitions between the windmill and propeller states.

First-order transfer functions are sometimes used to model the time lag associated with the unsteady aerodynamic response of wind turbines. The results obtained by WInDS indicate that this assumption is reasonable for above-rated operating conditions or low tip speed ratios. This assumption breaks down for high tip speed ratios and fails to address the spanwise induction minimum that was observed from the simulated wakes. Surprisingly, the first-order approach followed similar trends to the ITI Energy barge with platform pitching, independent of operating condition. This is likely due to the additional energy injected into the wake by the significant platform motions, indicating that while the first-order approach generated similar time lag responses, it was for the wrong reason.

Platform pitch drives the rotor thrust response for both the single and multiple-DOF test cases. Additionally, platform pitching appears to increase propeller state occurrence for outboard blade sections, particularly for above-rated conditions. The results obtained from these simulations indicate that the trailing hub and tip vortices have a significant influence on propeller state occurrence.

\section{Conclusions}

This study has identified various operating modes for OFWTs that may result in complex rotor-wake interactions. Platform motions will result in significant changes in induction over the rotor of an OFWT as compared to monopile. The interactions between the rotor and the wake, driven by platform motions, are of a complexity such that higher level aerodynamic analysis techniques like FVM may be necessary to accurately estimate wind turbine loads and performance. Additionally, designs that may be considered optimal via typical first-order approaches may be suboptimal once applied to the operating conditions of an OFWT. The effects of platform motions on OFWT aerodynamics are not insignificant and must be acknowledged. 


\section{Acknowledgments}

This research was supported by the University of Massachusetts Amherst Department for Mechanical and Industrial Engineering, the University of Massachusetts Amherst Wind Energy Center, and the Massachusetts Space Grant Consortium. Many thanks are also extended to J. Gordon Leishman of the University of Maryland and Friedemann Beyer of University of Stuttgart the for their assistance in this work.

\section{References}

1. Sebastian, T.; Lackner, M. Characterization of the unsteady aerodynamics of offshore floating wind turbines. Wind Energy 2012, doi: 10.1002/we.545.

2. Jonkman, J.; Butterfield, S.; Musial, W.; Scott, G. Definition of a 5-MW Reference Wind Turbine for Offshore System Development; Technical Report NREL/TP-500-38060; National Renewable Energy Laboratory: Golden, CO, USA, 2009.

3. Jonkman, J.M. Dynamics Modeling and Loads Analysis of an Offshore Floating Wind Turbine; Technical Report NREL/TP-500-41958; National Renewable Energy Laboratory: Golden, CO, USA, 2007.

4. Jonkman, J. Definition of the Floating System for Phase IV of OC3; Technical Report NREL/ TP-500-47535; National Renewable Energy Laboratory: Golden, CO, USA, 2010.

5. Matha, D. Model Development and Loads Analysis of an Offshore Wind Turbine on a Tension Leg Platform, with a Comparison to Other Floating Turbine Concepts; Technical Report NREL/ SR-500-45891; National Renewable Energy Laboratory: Golden, CO, USA, 2009.

6. IEC Technical Committee 88. Design Requirements for Offshore Wind Turbines: IEC 61300-3; Draft Standard; International Electrotechnical Commision: Geneva, Switzerland, 2010.

7. Jonkman, J.M.; Matha, D. Dynamics of offshore floating wind turbines-Analysis of three concepts. Wind Energy 2011, 14, 557-569.

8. Jonkman, J.M.; Buhl, M.L. Development and verification of a fully coupled simulator for offshore wind turbines preprint. In Proceedings of the 45th AIAA Aerospace Sciences Meeting and Exhibit, Reno, NV, USA, January 2007.

9. Moriarty, P.J.; Hansen, A.C. AeroDyn Theory Manual; Technical Report NREL/EL-500-36881; National Renewable Energy Laboratory: Golden, CO, USA, 2005.

10. Manjock, A. Design Codes FAST and ADAMS for Load Calculations of Onshore Wind Turbines; Technical Report Report No. 72042; Germanischer Lloyd WindEnergie GmbH: Hamburg, Germany, 2005.

11. Jonkman, J.; Sclavounos, P. Development of fully coupled aeroelastic and hydrodynamic models for offshore wind turbines. In Proceedings of the ASME Wind Energy Symposium, Reno, NV, USA, January 2006.

12. Sebastian, T.; Lackner, M. Development of a free vortex wake model code for offshore floating wind turbines. Renew. Energy 2011, 10.1016/j.renene.2012.03.033.

13. Bhagwat, M.J.; Leishman, J.G. Stability, consistency and convergence of time-marching free-vortex rotor wake algorithms. J. Am. Helicopter Soc. 2001, 46, 59-71. 
14. Leishman, J.G. Principles of Helicopter Aerodynamics (Cambridge Aerospace Series); Cambridge University Press: Cambridge, UK, 2006.

15. Bossanyi, E.A. GH Bladed Theory Manual; Garrad Hassan: London, UK, 2007.

16. Oye, S. Dynamic stall, simulated as time lag of separation. In Proceedings of the 4th IEA Symposium on the Aerodynamics of Wind Turbines, Rome, Italy, 20-21 November 1990.

(c) 2012 by the authors; licensee MDPI, Basel, Switzerland. This article is an open access article distributed under the terms and conditions of the Creative Commons Attribution license (http://creativecommons.org/licenses/by/3.0/.) 